NBER WORKING PAPER SERIES

\title{
WHAT IS THE PRICE OF TEA IN CHINA? TOWARDS THE RELATIVE COST OF LIVING IN CHINESE AND U.S. CITIES
}

\author{
Robert C. Feenstra \\ Mingzhi Xu \\ Alexis Antoniades \\ Working Paper 23161 \\ http://www.nber.org/papers/w23161 \\ NATIONAL BUREAU OF ECONOMIC RESEARCH \\ 1050 Massachusetts Avenue \\ Cambridge, MA 02138 \\ February 2017
}

We thank John Romalis and participants at an NBER conference for their helpful comments and Qi Liu for research assistance. Financial support from the National Science Foundation and the China Scholarship Council is gratefully acknowledged, as are the elasticities of substitution provided by Hottman, Redding, and Weinstein (2016). Calculations are based on data from the Nielsen Company (China) and from the Nielsen Company (US), LLC and marketing databases provided by the Kilts Center for Marketing Data Center at the University of Chicago Booth School of Business. Information about the U.S. data and access are available at http:// research.chicagobooth.edu/nielsen/. () The Nielsen Company. Circulated with permission. Please direct correspondence to: Robert Feenstra, University of California, Davis, rcfeenstra@ucdavis.edu. The views expressed herein are those of the authors and do not necessarily reflect the views of the National Bureau of Economic Research.

NBER working papers are circulated for discussion and comment purposes. They have not been peer-reviewed or been subject to the review by the NBER Board of Directors that accompanies official NBER publications.

(C) 2017 by Robert C. Feenstra, Mingzhi Xu, and Alexis Antoniades. All rights reserved. Short sections of text, not to exceed two paragraphs, may be quoted without explicit permission provided that full credit, including $\odot$ notice, is given to the source. 
What is the Price of Tea in China? Towards the Relative Cost of Living in Chinese and U.S.

Cities

Robert C. Feenstra, Mingzhi Xu, and Alexis Antoniades

NBER Working Paper No. 23161

February 2017

JEL No. E01,F11,L1

\begin{abstract}
$\underline{\text { ABSTRACT }}$
We examine the price and variety of products at the barcode level in cities within China and the United States. In both countries, there is a greater variety of products in larger cities. But in China, unlike the United States, the prices of products tend to be lower in larger cities. We attribute the lower prices to a pro-competitive effect, whereby large cities attract more firms which leads to lower markups and prices. Combining the effect of greater variety and lower prices, it follows that the cost of living for grocery-store products in China is lower in larger cities. We further compare the cost-of-living indexes for particular product categories between China and the United States. In product categories with a significant presence of U.S. brands in the Chinese market, the availability of additional Chinese brands leads to greater variety than in the United States, and therefore lower Chinese price indexes for that reason. In product categories with much less presence of U.S. brands in the Chinese market, however, the observed prices differences between the countries (usually lower prices in China) are partially or fully offset by the variety differences (less variety in China), so that the cost of living in China is not as low as the price differences suggest, especially in smaller cities.
\end{abstract}

Robert C. Feenstra

Department of Economics

University of California, Davis

One Shields Avenue

Davis, CA 95616

and NBER

rcfeenstra@ucdavis.edu

Mingzhi Xu

University of California, Davis

Department of Economics

mzhxu@ucdavis.edu
Alexis Antoniades

Georgetown University

aa658@georgetown.edu 


\section{Introduction}

In 2005, real GDP for the Chinese economy fell by $40 \%$. Not in reality, of course, but in the estimates reported by the World Bank. As explained by Deaton and Heston (2010), the revised 2005 estimates made use of new price data collected for China under the International Comparisons Program (ICP):

... the 2007 version of the World Development Indicators (WDI), World Bank (2007), lists 2005 per capita GDP for China as $\$ 6,757 \ldots$ in current international dollars. The 2008 version, World Bank (2008), which includes the new [2005] ICP data, gives, for the same year, and the same concept $\$ 4,088$ for China ... 1

The logical explanation for this fall in estimated real GDP for China is that the prices collected for China were higher than expected. In fact, prices had never been collected for China before the 2005 round of the ICP, so prior estimates of real GDP used imputed prices. Because the actual Chinese prices were higher than imputed, then the quantity of goods consumed by the representative Chinese individual were lower. It follows that real GDP was also lower - 40\% lower for China!

Two explanations have been provided for the unexpectedly high prices collected for China by the ICP 2005. The first explanation is that urban regions were over-sampled in China and that rural prices would be lower. That claim is evaluated by Feenstra, Ma, Neary, and Prasada Rao (2013), who find that Chinese prices from the 2005 ICP round were indeed somewhat higher than for other developing countries at similar levels of GDP per capita. Besides the possible urban bias, another explanation has to do with a special feature of the 2005 ICP, whereby prices were collected in each region of the world and then "linked" to other regions using a different price survey conducted only in certain cities. Deaton and Aten (2017) and Inklaar and Rao (2017) argue that this "linking" procedure led to a systematic upward bias in the prices for Asia relative to the United States. That linking procedure was not followed in the 2011 round of the ICP, and as a result, relative prices in Asia and in other developing countries were lower than in 2005.

These facts, combined with anecdotal evidence of high prices in China, ${ }^{2}$ are enough to convince us that a source of price information for China independent of the ICP is very important; crucial, in fact, to obtain reliable estimates of its real GDP. The goal of this paper is to compare the cost of living for cities in China and in the United States using two sources of barcode data: scanner data from grocery stores; and prices for grocery-store products scraped from a phone application. In scraping prices from a phone app we are following the lead of Cavallo and Rigobon (2016) who collect prices from internet sources to make time-series and cross-country comparisons. Such scraped data alone is not well-suited to compute cost-of-living indexes, however, because there is no quantity or expenditure data available with the scraped prices. While expenditure data is available from scanner data, it is prohibitively expensive to obtain for China in more than a handful of products.

\footnotetext{
${ }^{1}$ Deaton and Heston (2010), p. 3. The World Bank references in this quote are to the World Development Indicators.

${ }^{2}$ Anecdotal evidence that prices in China are high comes from Chinese students in the United States who return home and find a local price for an item that is higher than the U.S. price at the official exchange rate. They will photograph that item and post it on their Facebook page!
} 
Accordingly, in this paper we rely on Chinese scanner data for four product categories in 22 cities, purchased from Nielsen (China), and have supplemented these data with scraped prices for these same four products and a further 15 product categories over 60 cities, obtained from a mobile phone application that allows consumers to check for the various prices at supermarkets in each city. These Chinese price data are complemented by Nielsen (U.S.) barcode data at the city level. All data are for 2011 or more recent years.

In our results, we find that in both countries there is a greater variety of products in larger cities. That result has already been found by Handbury and Weinstein (2015) for the United States, and this paper is the first confirmation of the same result for China. For the U.S., the greater variety in larger cities offsets the higher prices found there, so that Handbury and Weinstein (2015) argue that the cost of living - incorporating both price and variety - is lower in larger cities. But in China, unlike the United States, we find that the prices of products themselves tend to be lower in larger cities. We attribute those lower prices to a pro-competitive effect, whereby large cities attract more firms which leads to lower markups and prices. Combining the effect of greater variety and lower prices, it follows that the cost of living for grocery-store products in China is also lower in larger cities.

We further compare the cost-of-living indexes for particular products between China and the United States. In the four product categories for which we purchased Nielsen (China) scanner data, i.e., Toothpaste, Laundry Detergent, Personal Wash items and Shampoo, we expected a significant presence of U.S. brands in the Chinese market and that turned out to be the case. Then the availability of additional Chinese brands leads to greater variety than in the United States, and therefore lower Chinese price indexes for that reason. In the other 15 product categories for which we only have scraped data, however, there is much less presence of U.S. brands in the Chinese market. In these cases, the observed prices differences between the countries (usually lower prices in China) are partially or fully offset by the variety differences (less variety in China), so that the cost of living in China is not as low as the price differences suggest. This latter case applies to Tea, for example, because our data do not include the many Chinese varieties of tea that are not sold in supermarkets.

In section 2 we describe the nested CES framework that we shall use to measure the consumer gains from variety. Preliminary evidence from the four product categories with both Nielsen (China) barcode data and scanned prices is presented in section 3. A model of multi-product firms is described in section 4, from which we derive the solutions for firm pricing and product scope. These equations are estimated as regressions described in section 5. Our empirical results on the prices of 15 other product categories is presented in section 6 , and section 7 concludes.

\section{Consumer Utility and Variety}

We study an economy consisting of $c=1, \ldots, D$ cities or destinations, which differ in population $L_{c}$ and labor income $w_{c}$. Labor is the only factor of production and it is not mobile across cities. A fraction $\rho$ of total labor income is spent on the differentiated goods, and we denote that spending by 
$Y_{c}=\rho w_{c} L_{c}$.

The preferences of the representative consumer in each city are nested CES. Denoting the set of product varieties sold by firm $f$ in city $c$ by $i \in I_{f c}$, the sub-utility from the products of firm $f$ are given by

$$
X_{f c}=\left(\sum_{i \in I_{f c}}\left(b_{f i c} x_{f i c}\right)^{(\sigma-1) / \sigma}\right)^{\sigma /(\sigma-1)}, \sigma>1,
$$

where $\sigma$ is the elasticity of substitution across products sold by a firm, and $b_{\text {fic }}$ are the taste parameters for each variety, which we will allow to differ across firms, products and to a certain extent across cities as explained below. Each firm is a manufacturing firm and not a retailer. ${ }^{3}$ Aggregating across firms $f \in F_{c}$ that sell in city $c$, utility of the representative consumer is,

$$
U_{c}=\left(\sum_{f \in F_{c}} X_{f c}^{(\eta-1) / \eta}\right)^{\eta /(\eta-1)}, \eta>1 .
$$

As in Hottman, Redding, and Weinstein (2016), we expect that the elasticity of substitution $\sigma$ across products within a firm is larger than the elasticity $\eta$ across firms, so we assume that $\sigma \geq \eta>1$. When the two elasticities are equal, then the nested CES system will collapse to a standard CES utility function as used in Feenstra (1994).

Let $\mathbf{p}_{f c}$ denote the vector of prices $p_{f i c}$ for firm $f$ across all product varieties $i$, with the vector of taste parameters $\mathbf{b}_{f c}$, and let $P_{f_{c}}=e\left(\mathbf{p}_{f c}, \mathbf{b}_{f_{c}}, I_{f_{c}}\right)$ denote the minimum expenditure needed to obtain one unit of sub-utility, $X_{f_{c}}=1$. Then $e\left(\mathbf{p}_{f_{c}}, \mathbf{b}_{f c}, I_{f_{c}}\right)$ takes on the CES form,

$$
P_{f_{c}}=e\left(\mathbf{p}_{f c}, \mathbf{b}_{f c}, I_{f_{c}}\right)=\left(\sum_{i \in I_{f c}}\left(p_{f i c} / b_{f i c}\right)^{1-\sigma}\right)^{1 /(1-\sigma)} .
$$

Feenstra (1994) shows how to measure the effect of new varieties on the exact price index. Specifically, consider firm $f$ selling to destinations $c$ and $d$. Suppose that there is a non-empty subset of "common" products $I_{f} \subseteq I_{f c} \cap I_{f d}$ sold by firm $f$ in these two cities for which the taste parameters are equal, $b_{f i c}=b_{f i d}, i \in I$. Then the exact price index between the two cities can be expressed as

$$
\frac{e\left(\mathbf{p}_{f c}, \mathbf{b}_{f c}, I_{f c}\right)}{e\left(\mathbf{p}_{f d}, \mathbf{b}_{f d}, I_{f d}\right)}=\left[\prod_{i \in I_{f}}\left(\frac{p_{f i c}}{p_{f i d}}\right)^{w_{f i}\left(I_{f}\right)}\right]\left(\frac{\lambda_{f c}}{\lambda_{f d}}\right)^{\frac{1}{\sigma-1}} .
$$

The first term in brackets on the right of (4) is the Sato (1976)-Vartia (1976) price index, where $w_{f i}\left(I_{f}\right)$ is the weight defined by:

$$
w_{f i}\left(I_{f}\right) \equiv \frac{\frac{s_{f i c}\left(I_{f}\right)-s_{f i d}\left(I_{f}\right)}{\ln s_{f i c}\left(I_{f}\right)-\ln s_{f i d}\left(I_{f}\right)}}{\sum_{j \in I_{f}}\left(\frac{s_{f j c}\left(I_{f}\right)-s_{f j d}\left(I_{f}\right)}{\ln s_{f j c}\left(I_{f}\right)-\ln s_{f j d}\left(I_{f}\right)}\right)}, \quad s_{f i c}\left(I_{f}\right) \equiv \frac{p_{f i c} x_{f i c}}{\sum_{j \in I_{f}} p_{f j c} x_{f j c}},
$$

\footnotetext{
${ }^{3}$ We examined the modeling of a retail sector as another nest in the utility function, with imperfectly competitive retailers. While the theory can be developed, we do not have any data on sales outside of the major retailer chains that we could use to calibrate this aspect of the model. So that extension is not considered here.
} 
and likewise for the shares $s_{\text {fid }}\left(I_{f}\right)$ in city $d$, also defined over the common set of products. The second term on the right of (4) is the adjustment needed to take into account differing sets of goods available in the two cities, and is defined by:

$$
\lambda_{f c} \equiv \frac{\sum_{i \in I_{f}} p_{f i c} x_{f i c}}{\sum_{i \in I_{f c}} p_{f i c} x_{f i c}}=1-\frac{\sum_{i \in I_{f c} \backslash I_{f}} p_{f i c} x_{f i c}}{\sum_{i \in I_{f c}} p_{f i c} x_{f i c}},
$$

To interpret these formulas, $\lambda_{f c}$ in (6) denotes the spending in city $c$ on the common products of firm $f$, sold in both cities, relative to total spending in city $c$ on firm $f^{\prime}$ s products. Equivalently, it equals one minus the share of expenditure on the unique products sold by firm $f$ only in city $c$. Having access to more unique varieties in city $c$ implies a smaller expenditure share on common products, $\lambda_{f c}$, and a lower cost-of-living index in (4).

To extend the exact price index to the nested CES case, let $\mathbf{P}_{c}$ denote the vector of CES price indexes $P_{f_{c}}$ shown in (3) for all firms $f \in F_{c}$ in city $c$, and let $P_{c}=E\left(\mathbf{P}_{c}, F_{c}\right)=\left(\sum_{f \in F_{c}} P_{f c}^{(\eta-1)}\right)^{1 /(1-\eta)}$ denote the expenditure needed to obtain utility of one in city . Let $F \equiv F_{c} \cap F_{d}$ denote the non-empty set of "common" firms selling to both cities $c$ and $d$. Then again from Feenstra (1994), the cost of living between the two cities can be written as,

$$
\begin{aligned}
\frac{E\left(\mathbf{P}_{c}, F_{c}\right)}{E\left(\mathbf{P}_{d}, F_{d}\right)} & =\left[\prod_{f \in F}\left(\frac{P_{f c}}{P_{f d}}\right)^{W_{f}(F)}\right]\left(\frac{\lambda_{c}}{\lambda_{d}}\right)^{\frac{1}{\eta-1}} \\
& =\left[\prod_{f \in F} \prod_{i \in I_{f}}\left(\frac{p_{f i c}}{p_{f i d}}\right)^{W_{f}(F) w_{i}\left(I_{f}\right)}\right]\left[\prod_{f \in F}\left(\frac{\lambda_{f c}}{\lambda_{f d}}\right)^{W_{f}(F)}\right]^{\frac{1}{\sigma-1}}\left(\frac{\lambda_{c}}{\lambda_{d}}\right)^{\frac{1}{\eta-1}}
\end{aligned}
$$

where the Sato-Vartia weights across firms are,

$$
W_{f}(F) \equiv \frac{\frac{S_{f c}(F)-S_{f d}(F)}{\ln S_{f c}(F)-\ln S_{f d}(F)}}{\sum_{g \in F}\left(\frac{S_{g c}(F)-S_{g d}(F)}{\ln S_{g c}(F)-\ln S_{g d}(F)}\right)}, \quad S_{f c}(F) \equiv \frac{\sum_{i \in I_{f c}} p_{f i c} x_{f i c}}{\sum_{g \in F} \sum_{i \in I_{g c}} p_{g i c} x_{g i c}},
$$

and likewise for the shares $S_{f d}(F)$ for city $d$, also defined over the common set of firms. The final term on the right of (7) is defined by:

$$
\lambda_{c} \equiv \frac{\sum_{g \in F} \sum_{i \in I_{g c}} p_{\text {gic }} x_{\text {gic }}}{\sum_{g \in F_{c}} \sum_{i \in I_{g c}} p_{\text {gic }} x_{\text {gic }}}=1-\frac{\sum_{g \in F_{c} \backslash F} \sum_{i \in I_{g c}} p_{\text {gic }} x_{\text {gic }}}{\sum_{g \in F_{c}} \sum_{i \in I_{g c}} p_{\text {gic }} x_{\text {gic }}},
$$

That is, $\lambda_{c}$ denotes the spending on the common set of firms $F$ relative to total spending in city $c$, or one minus the share of spending on firms selling only in city $c$. The greater the share of spending on unique firms selling only in that city, the lower is the exact price index in (7). 


\section{Evidence on Prices in Four Product Categories}

\subsection{Data}

Our calculation of the cost of living relies on the data extracted from three sources. The first source is the Nielsen (China) Sales database which enables us to observe the annual sales and average price information of each product with a barcode. ${ }^{4}$ We purchased Nielsen (China) scanner data for four product categories, namely, Toothpaste, Laundry Detergent, Personal Wash items, and Shampoo, covering 22 cities in China, as shown in the red colored regions of Figure $1 .{ }^{5}$ Besides the sales information for each product, we also observe manufacturer information such as brand and sub-brand of each product. In our theory and empirical analysis, we refer to the brand as the firm (e.g. Crest or Colgate for toothpaste).

The regions covered by Nielsen (China) database are only large cities (most of them are capital cities). To address this limitation, our analysis also relies on our second data source, which are scraped prices collected in 2015 from a mobile phone application that allows consumers to check for the various prices at supermarkets in each city. Details on this second source of data are provided in Appendix, and it allows us also to include smaller cities in our sample (as shown in Figure 11 of Appendix A3). We end up expanding our sample to 60 cities, including the 22 cities provided by Nielsen (China) database, as shown in the yellow colored regions of Figure 1. Based on the first two data sources, we implement the formulas described above to calculate the cost of living in China for each of the four product categories. Our third source of data is the Nielsen Retailing Sales (U.S.) database, used to calculate the cost of living for 377 MSAs of the United States in 2013, for each of the four product categories.

We have prices for individual barcode products in four categories for 60 cities in China, but we have expenditure on these barcode products for only 22 cities in the Nielsen (China) database. We have implemented a Heckman procedure to estimate barcode-level expenditure in the remaining 38 cities, using a reduced-form equation for the barcode expenditure shares in the 22 cities for which we have those data. This Heckman procedure is described in Appendix B. The estimated expenditure shares are used to measure the terms $\lambda_{f c}$ and $\lambda_{f}$ appearing in the cost-of-living index (7).

Data are also needed on the elasticities $\sigma$ between products within a firm, and $\eta$ between firms. These elasticities of substitution differ across the four product categories. We rely on the estimates of these elasticities from Hottman, Redding, and Weinstein (2016), as shown in Appendix B. These authors estimate the elasticities from the Nielsen Retailing Sales (U.S.) database, which is the same data that we use for the United States. Our assumption is that, for each product category, the elasticities are the same in the United States and in China.

\footnotetext{
${ }^{4}$ We convert RMB data to U.S. dollars using the annual average exchange rate.

${ }^{5}$ We use 2011 and 2012 sales information for toothpaste, and 2014 for the other three product categories.
} 


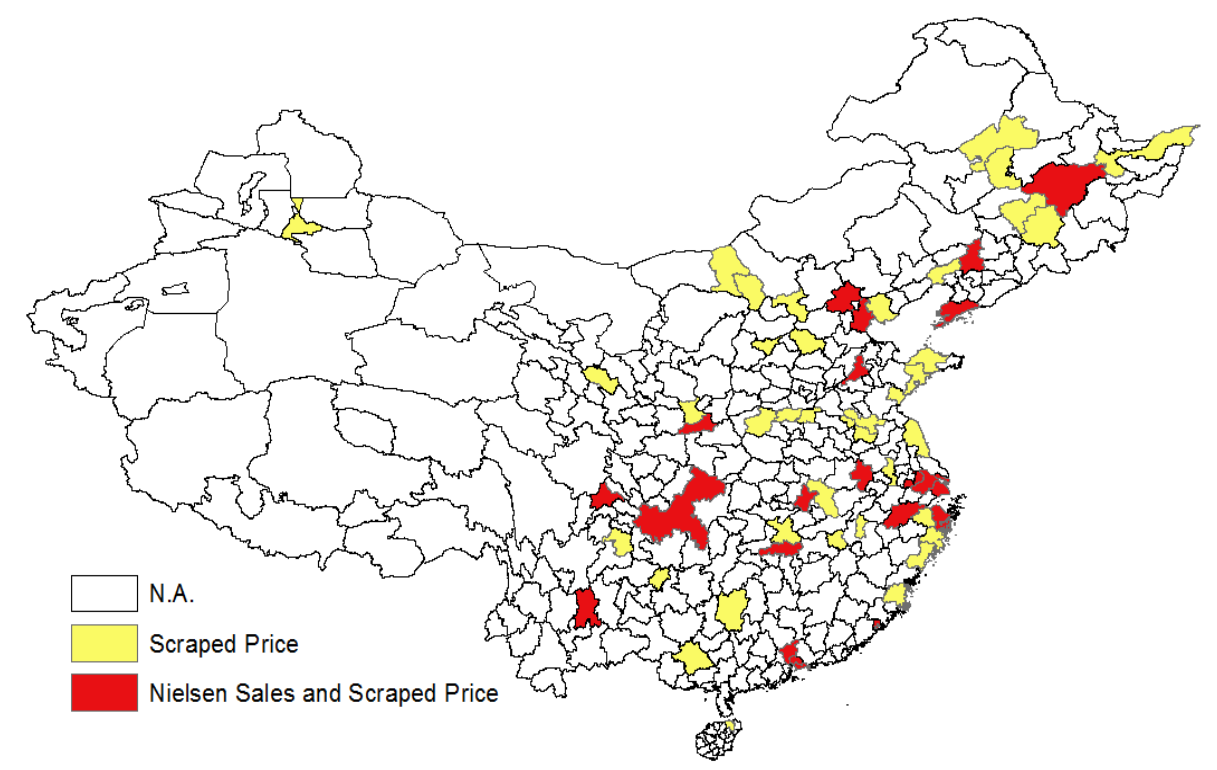

Figure 1: Regions included in Nielsen Sales Data and Scraped Price Data

Significantly, the barcode systems used in China is the European Article Number (EAN-13), whereas that used in the United States in the Universal Product Code (UPC). While it is not difficult to identify similar product categories, it is impossible to exactly match the items across countries by their barcodes since the barcode systems differ and product descriptions themselves do not match. That difference in the barcode systems means that the consumer theory outlined above due to Feenstra (1994) cannot be applied between countries, since it assumed that there is a non-empty subset of "common" products $I_{f} \subseteq I_{f c} \cap I_{f d}$ sold by firm $f$ in two cities $c$ and $d$ for which the taste parameters are equal, $b_{f i c}=b_{f i d}, i \in I$. We certainly cannot assume that the tastes for products are the same when we cannot even match products across barcode systems. Accordingly, for the next several sections we focus on comparisons of price across cities within China and the U.S. It will not be until section 6 that we attempt to compare across countries, using an alternative formulation of exact price indexes for a nested CES utility function due to Redding and Weinstein (2016).

\subsection{Prices Across Chinese and U.S. Cities}

Panel (a) of Figures 2 to 5 exhibits how the average price of the common products vary with respect to the city population for Toothpaste, Laundry Detergent, Personal Wash Items and Shampoo. We measure the prices in terms of dollars per ounce and calculate the average price using the Sato-Vartia weights, which is the numerator of the Sato-Vartia index in (7), while also calculating the numerator of the variety adjustment in (7): 


$$
P_{c}^{S V} \equiv \prod_{f \in F} \prod_{i \in I_{f}}\left(p_{f i c}\right)^{W_{f}(F) w_{i}\left(I_{f}\right)}, \quad \Lambda_{c}^{S V} \equiv\left[\prod_{f \in F} \lambda_{f c}^{W_{f}(F)}\right]^{\frac{1}{\sigma-1}}\left(\lambda_{c}\right)^{\frac{1}{\eta-1}}
$$

The terms $P_{c}^{S V}$ and $\Lambda_{c}^{S V}$ are shown in panels (a) and (b) of Figure 2 for Toothpaste, Figure 3 for Laundry Detergent, Figure 4 for Personal Wash items, and Figure 5 for Shampoo. In each panel and each Figure, we plot the variables for the United States (in blue dots) and China (in red x) against the log population of each city.

From panel (a), we observe that average prices $P_{c}^{S V}$ of common products within the United States do not vary significantly between cities, whereas the average prices in China have a clear negative relationship with city size. We explore that finding more in the next section, where we argue that a pro-competitive effect in larger cities in China can account for that result. In panel (b) we see that the term $\Lambda_{c}^{S V}$, which measures the inverse of variety, falls slightly with city size in the United States (though this is barely visible), but falls very noticably with city size in China.

Multiplying the terms $P_{c}^{S V} \times \Lambda_{c}^{S V}$, and choosing the normalization $P_{N Y}^{S V} \Lambda_{N Y}^{S V}=1$ for New York, we obtain the exact price index for the United State shown in panel (c) of each Figure. These exact price indexes are slightly declining in city size for Toothpaste, Laundry Detergent, and Personal Wash items, but not for Shampoo. In the first three products, we therefore confirm the finding by Handbury and Weinstein (2015) for the United States that the greater variety in larger cities offsets the higher prices found there. But in China, unlike the United States, the prices of products themselves tend to be lower in larger cities, and that is reinforced by a much greater increase in variety in larger cities, too. For both reasons, the cost-of-living indexes $P_{c}^{S V} \Lambda_{c}^{S V}$ for China decline much more rapidly with population than in the United States.

Because the terms in (10) use the common products within each country, these variables are only meaningful when comparing prices and variety for each country. In section 6 we develop a method for comparing these indexes between countries, drawing on Redding and Weinstein (2016). Continuing to normalize $P_{N Y}^{S V} \Lambda_{N Y}^{S V}=1$ for New York, we apply this between-country method to obtain the exact prices index for Shanghai, abbreviated as the point $\mathrm{SH}$ in panel (c). Then the indexes $P_{c}^{S V} \Lambda_{c}^{S V}$ for all other Chinese cities are measured relative to Shanghai and, by of the virtue of Redding and Weinstein (2016) method described in section 6, these can also be compared to those in the U.S. We see that the cost of living for each product in panel (c) is pulled down in China as compared to the Sato-Vartia geometric means in panel (a), especially in the larger Chinese cities. In other words, for all four product categories, the additional product variety (as shown in panel b) due to both U.S. and local brands in the larger cities in China lowers the relative cost of living for consumers there. 

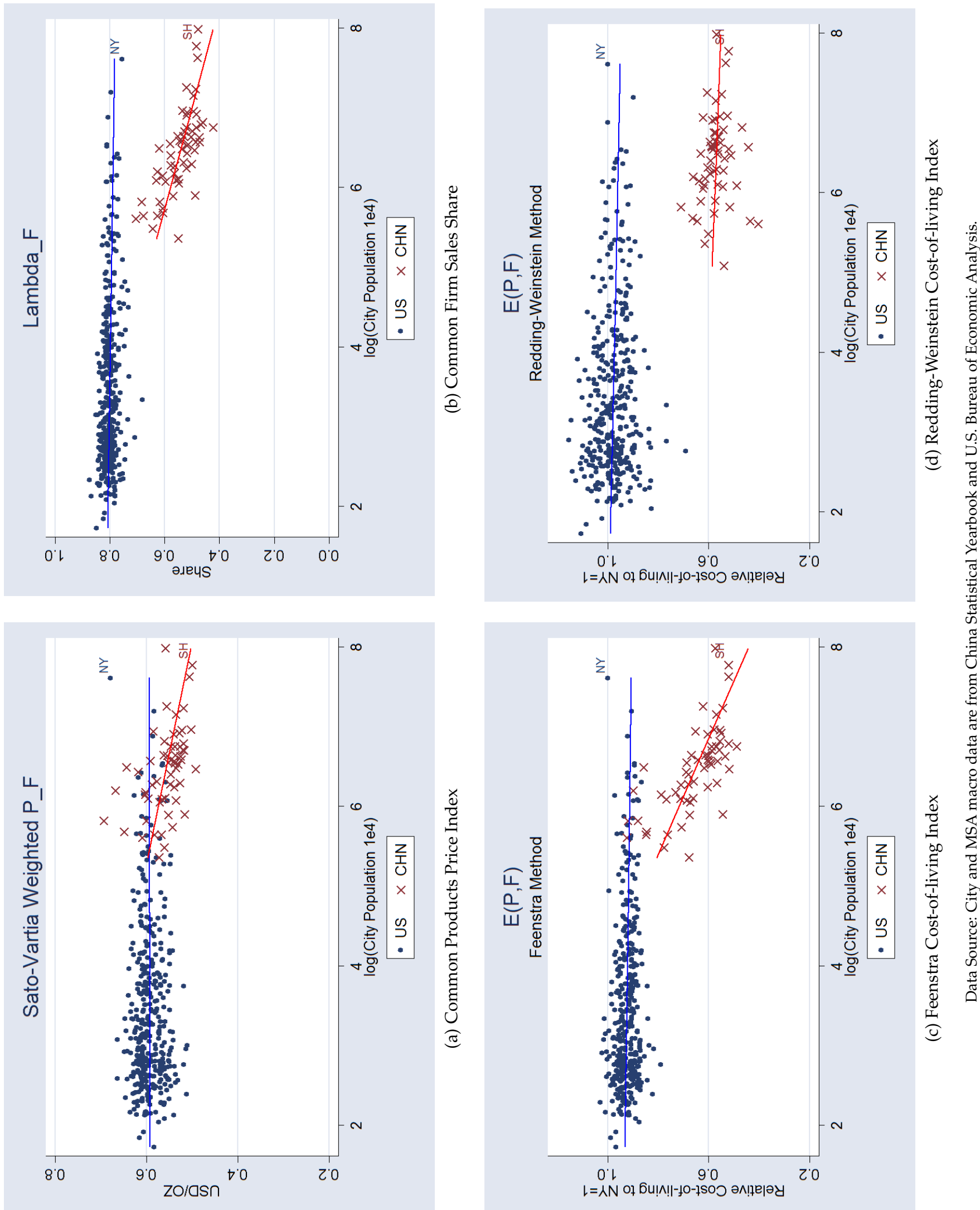

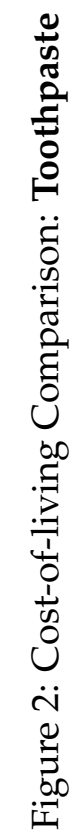



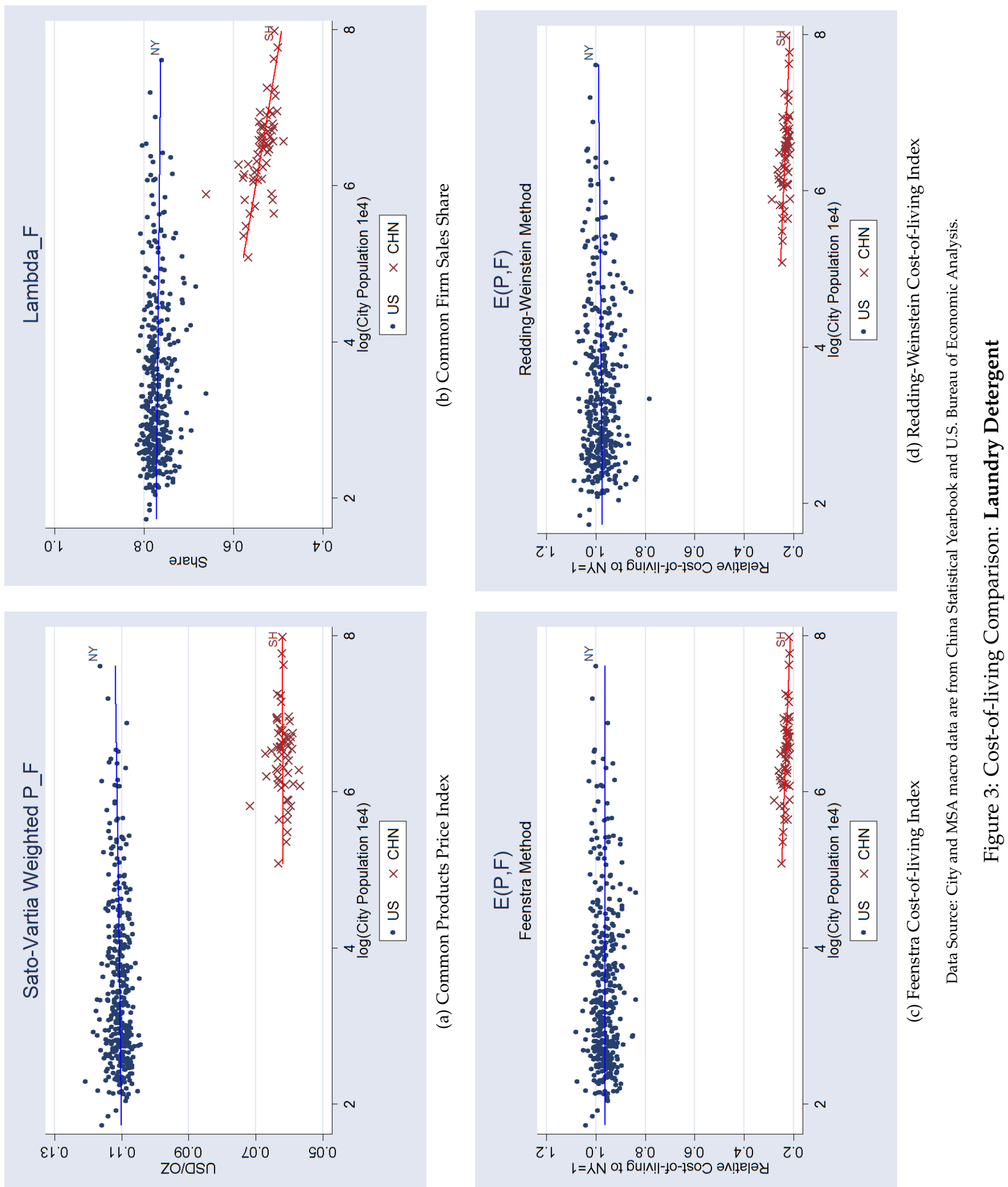

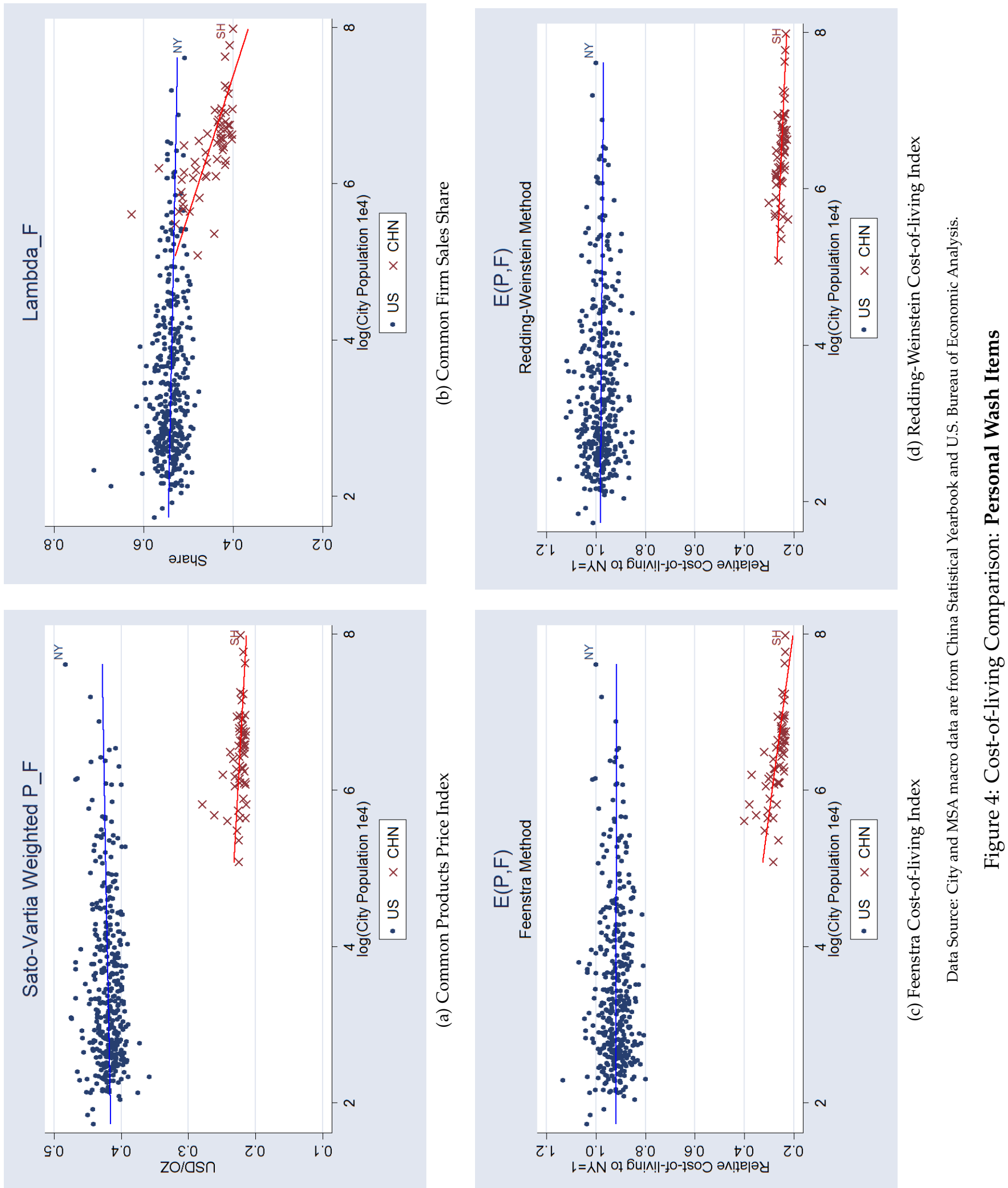

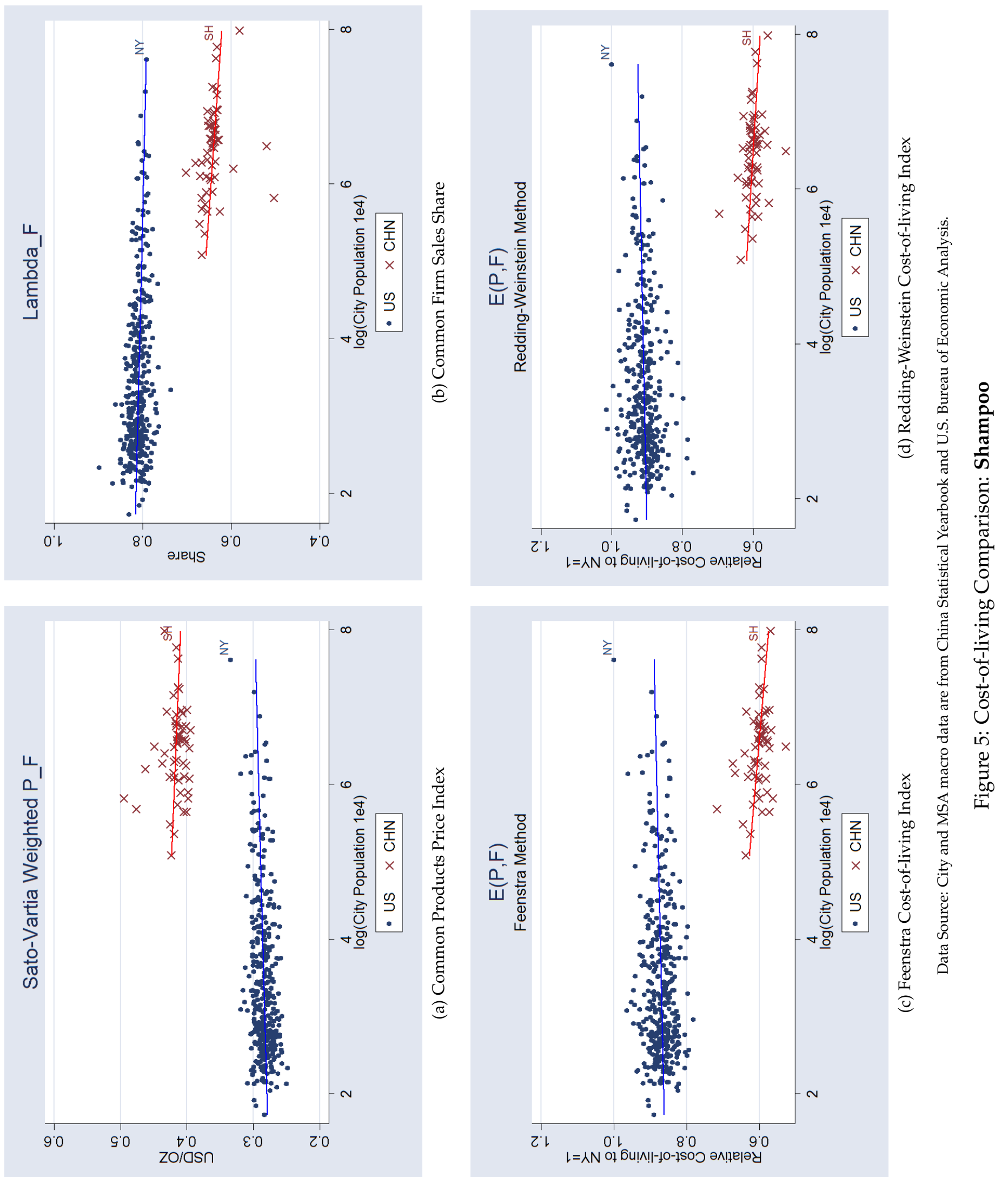
We further illustrate the cost of living in each Chinese city relative to the average level of cost of living in the United States, ${ }^{6}$ by plotting these in maps shown in Figures 6 to 9. We use the cold color (blue) to indicate a Chinese price level lower than that of the U.S. average, and the hot color (red) to represent a higher Chinese price level. We see from Figure 6 to 9 that the exact price index is lower in China than the U.S. in all four product categories (the relative price varies from 0.24 to 0.71, with the U.S. average as unity). This occurs despite the fact that the Sato-Vartia average price for Shampoo is higher in China than in the United States. ${ }^{7}$ In addition, the exact price indexes are higher in inland cities than in the eastern coast cities that are usually of larger size. For example, the most expensive cities for Toothpaste, Laundry Detergent, Personal Wash items, and Shampoo are Baotou (1.00), Lanzhou (0.29), Baotou (0.44), and Hohhot (0.83) respectively. So the benefits of greater variety in China applies only to the larger cities, often found on the eastern coast and in certain inland locations such as Chongching, which is highlighted in the maps near the center of China and has relatively low exact price indexes.

\section{Firm Pricing and Choice of Variety}

\subsection{Nested CES Demand}

To obtain the demand for each differentiated good consumed in country $c$, let us start at the firm level. Demand for the aggregate of firm $f$ products is $X_{f_{c}}=\left(Y_{c} / P_{c}\right)\left(P_{f_{c}} / P_{c}\right)^{-\eta}$, where $Y_{c}$ denotes total expenditure, $P_{c}$ is the overall CES price index, and $P_{f_{c}}$ is the CES price index (or unit-expenditure function) shown in (3). Then demand for each variety equals $b_{f i c} x_{f i c}=\left[\left(p_{f i c} / b_{f i c}\right) / P_{f c}\right]^{-\sigma} X_{f c}$. Multiplying by $\left(p_{f i c} / b_{f i c}\right)$ and using the equation for $X_{f c}$, we find that spending on each variety is,

$$
p_{f i c} x_{f i c}=P_{f c} X_{f c}\left(\frac{p_{f i c} / b_{f i c}}{P_{f c}}\right)^{1-\sigma}=Y_{c}\left(\frac{p_{f i c} / b_{f i c}}{P_{f c}}\right)^{1-\sigma}\left(\frac{P_{f c}}{P_{c}}\right)^{1-\eta} .
$$

We are now in a position to compute the elasticity of demand for an individual variety. We use the log of spending and differentiate to obtain:

$$
\begin{aligned}
\varepsilon_{f i c} & =-\frac{d \ln x_{f i c}}{d \ln p_{f i c}}=1-\frac{d \ln \left(p_{f i c} x_{f i c}\right)}{d \ln p_{f i c}} \\
& =1-(1-\sigma)-\left[(\sigma-\eta)+(\eta-1) \frac{d \ln P_{c}}{d \ln P_{f c}}\right] \frac{d \ln P_{f c}}{d \ln p_{f i c}} \\
& =\sigma-\left[(\sigma-\eta)+(\eta-1) S_{f c}\right] s_{f i c}
\end{aligned}
$$

where $s_{f i c}=d \ln P_{f c} / d \ln p_{f i c}$ is the share of expenditure on product $i$ within the sales of firm $f$, and $S_{f c}=d \ln P_{c} / d \ln P_{f c}$ is the total share of sales of firm $f$ in city $c{ }^{8}$

${ }^{6}$ The U.S. average cost of living is calculated as the geometric mean of all MSAs.

${ }^{7}$ We suspect that shampoo products in China are more likely to include additives that raise their prices.

${ }^{8}$ Using our notation of section 2, the within-firm share of expenditure is $s_{f i c}=s_{f i c}\left(I_{f c}\right)$, and the firm total share of 


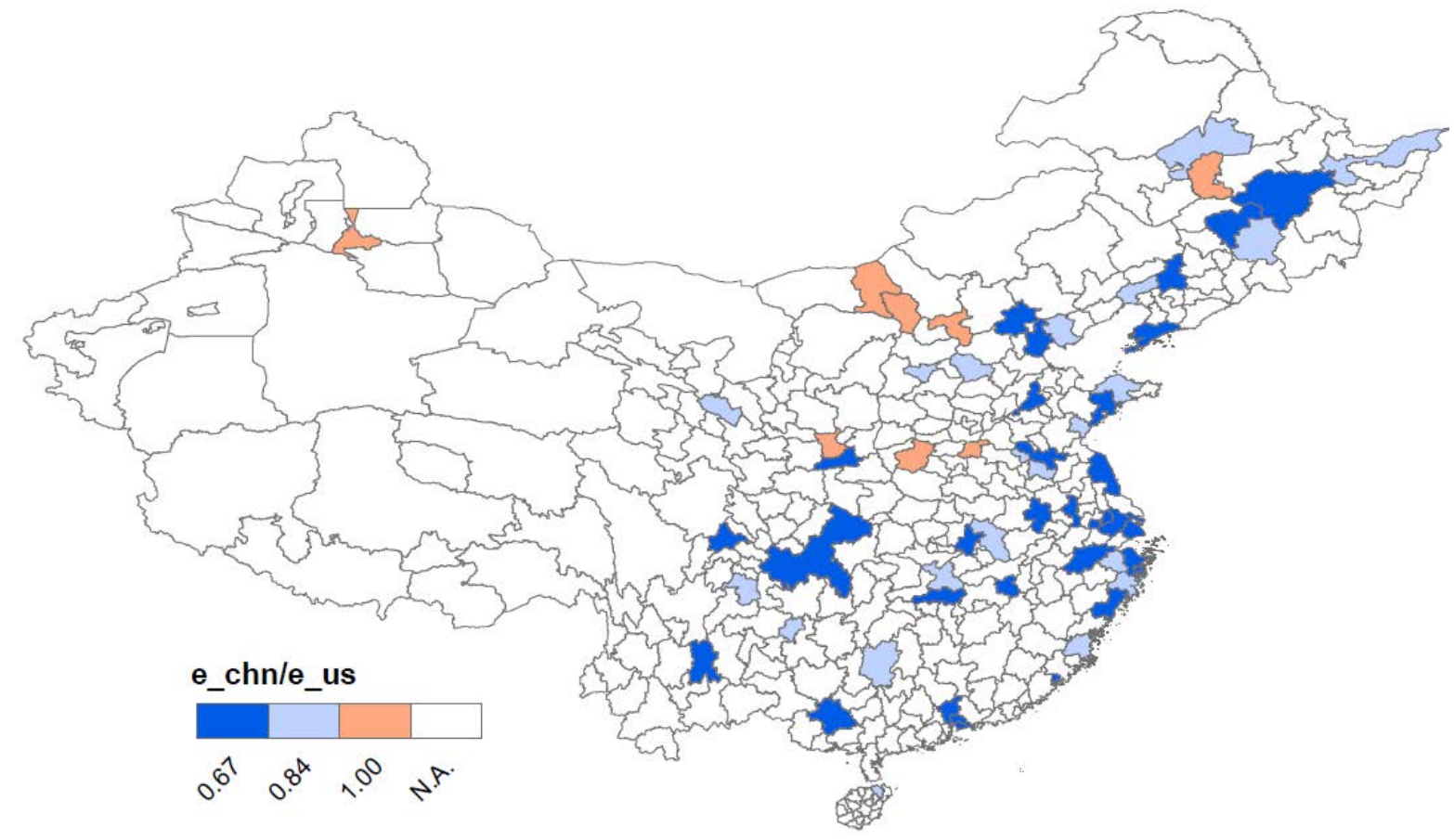

Figure 6: Relative Cost-of-living in China: Toothpaste

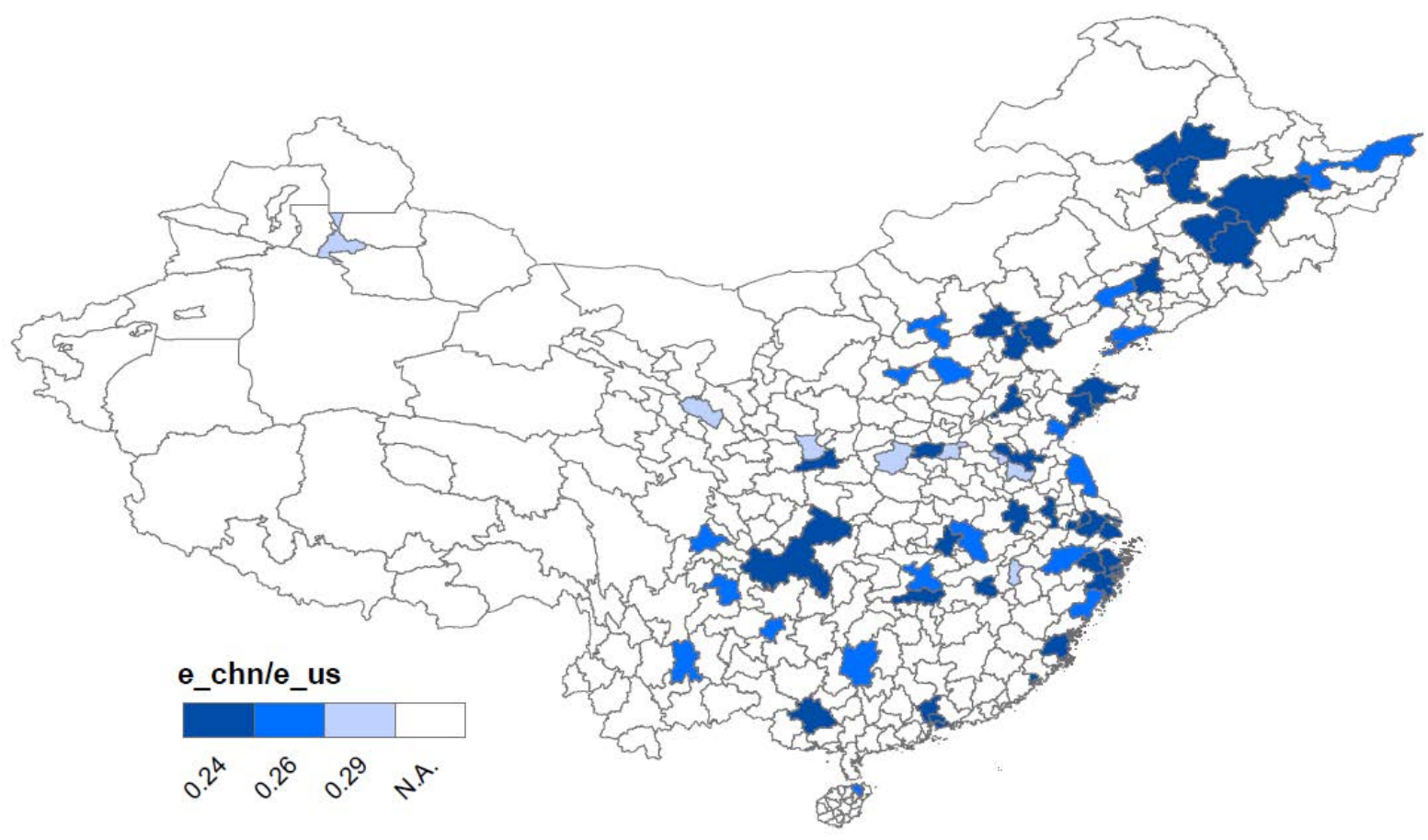

Figure 7: Relative Cost-of-living in China: Laundry Detergent

expenditure is $S_{f_{c}}=S_{f_{c}}\left(F_{c}\right)$. 


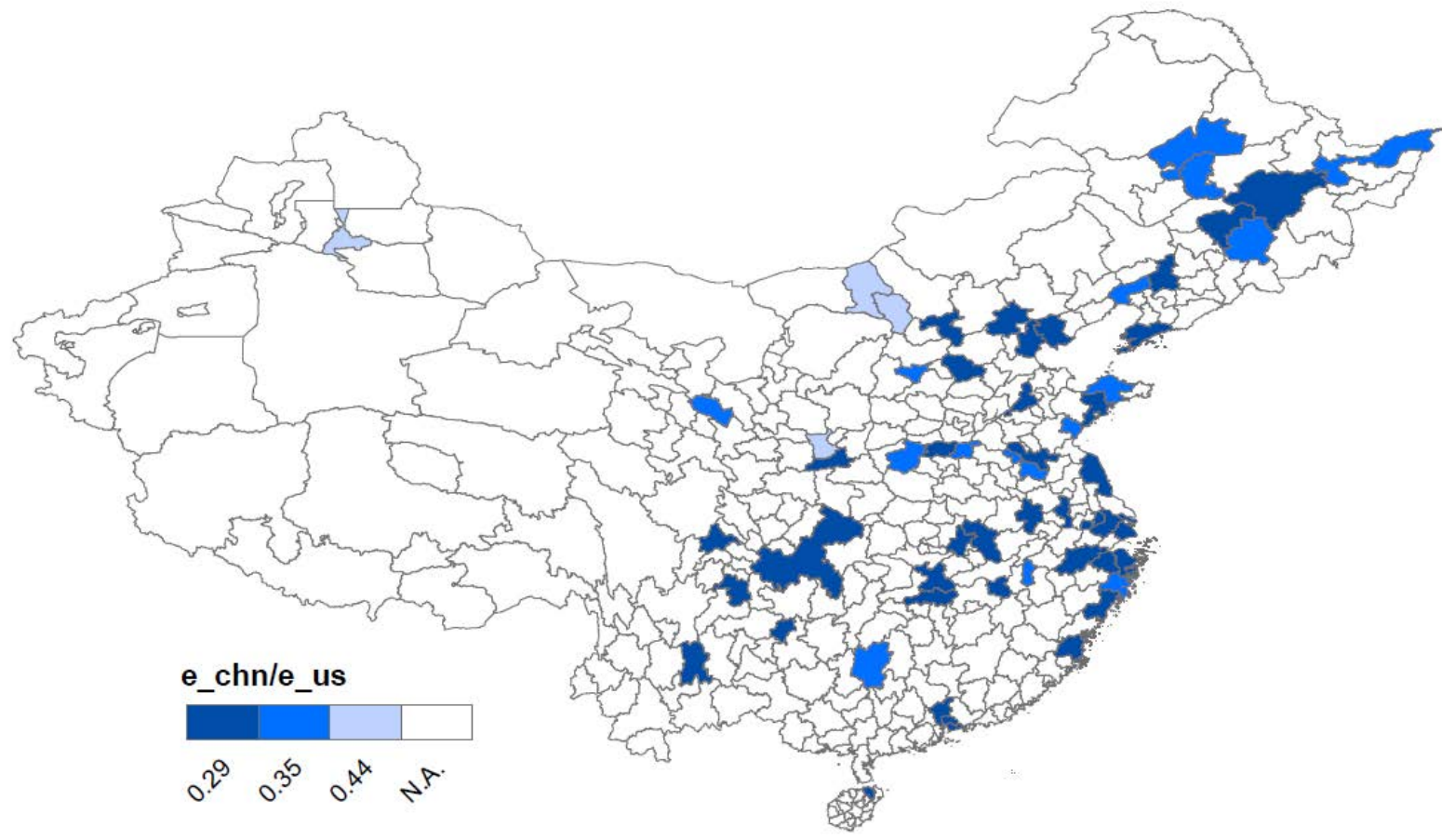

Figure 8: Relative Cost-of-living in China: Personal Wash Items

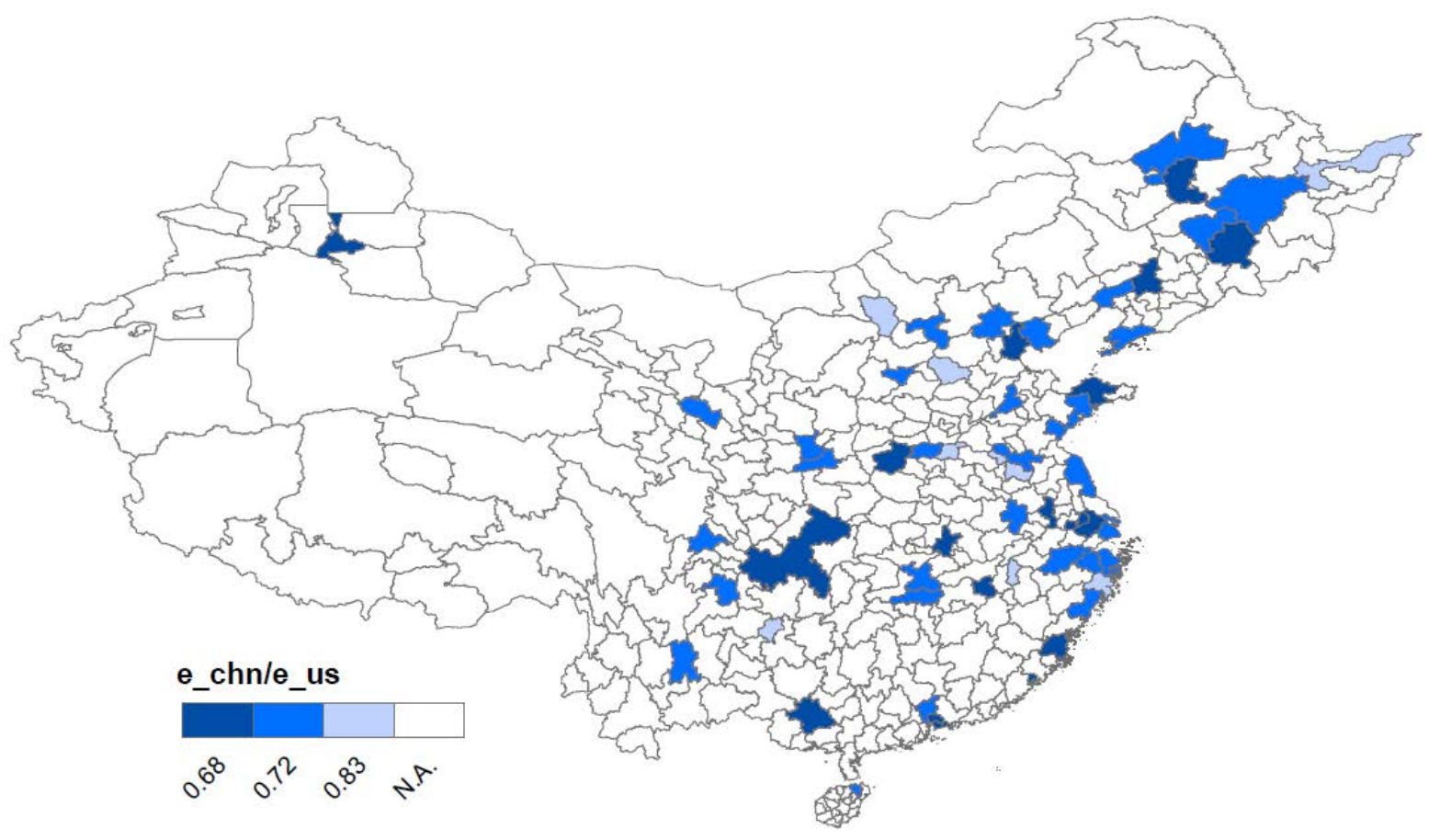

Figure 9: Relative Cost-of-living in China: Shampoo 
Our assumption that $\sigma \geq \eta$ implies that as the variety share $s_{f i c}$ rises then the elasticity of demand will fall; likewise, as the firm share $S_{f_{c}}$ rises the elasticity also falls. If the firm ignored the cannibalization effect of each variety on other sales then it would charge a higher price whenever $s_{f i c}$ or $S_{f c}$ rises. But we now show that when the firm jointly profit-maximizes over all goods, taking into account cannibalization effects, then the price charged for each good will depend only the firm share $S_{f c}$ and not on the within-firm variety share $s_{f i c}$.

\subsection{Optimal Prices for the Multiproduct Firm}

Consider a firm producing variety $i$ in city $c$ and delivering it to destination $d$. The firm chooses the range of products to sell in multiple destinations $d=1, \ldots D$. The profit-maximization problem for this firm is

$$
\max _{p_{f i d}, i \in I_{f d}} \sum_{d=1}^{D}\left\{\left[\sum_{i \in I_{f d}}\left(p_{f i d}-g_{f i}\left(w_{c}\right)-T_{c d}\right) x_{f i d}-k_{f i d}\right]-K_{f d}\right\} \mathbf{1}\left(I_{f d} \neq \varnothing\right),
$$

where $K_{f d}$ denotes the fixed costs to enter a city and $\mathbf{1}\left(I_{f d} \neq \varnothing\right)$ is an indicator variable that takes value of unity if $I_{f d} \neq \varnothing$ and zero otherwise. That is, the firm chooses whether to enter into each city $d$, and pays the fixed cost of $K_{f d}$ only if it does, along with the fixed costs of $k_{f i d}$ to sell each variety $i$ in city $d$. We let $g_{f i}\left(w_{c}\right)$ denote the (constant) marginal costs of producing good $i$ in city $c$, with factor prices $w_{c}$, and selling it in city $d$ with transport costs of $T_{c d}$. We assume that firms treat the prices of other firms as given under Bertrand competition, and that demand in the various cities is independent.

Focus initially on the choice of optimal prices. If the firm sold only a single product $i$ in destination $d$, so that $s_{f i d}=1$, then the elasticity of demand from (12) is $\epsilon_{f i d}=\eta-(\eta-1) S_{f d}$ so that $\epsilon_{f i d}-1=(\eta-1)\left(1-S_{f d}\right)$. It follows from the usual markup formula that the optimal price is,

$$
p_{f i d}=\left[1+\frac{1}{(\eta-1)\left(1-S_{f d}\right)}\right]\left[g_{f i}\left(w_{c}\right)+T_{c d}\right]
$$

When the firm sells multiple products, however, then it must take into account how a reduction in the price of one will decrease demand for its other products: this is the cannibalization effect. Nevetheless, we show in Appendix C that the same pricing formula as in (14) is obtained. ${ }^{9}$ In other words, when the firm jointly maximizes over all its prices, the markup obtained is "as if" it was using the elasticity of demand in (12) but with $s_{f i d}=1$, so that the markup depends only on the total market share $S_{f d}$ of the firm in that city.

\subsection{Optimal Variety for the Multiproduct Firm}

For simplicity, suppose that in each city there is symmetric demand and marginal cost for the products of each firm, ${ }^{10}$ and that the firm sells $N_{f d}$ of these varieties in city $d$. We allow marginal and

\footnotetext{
${ }^{9}$ This result is also shown by Feenstra (2016), chapter 9, and Hottman, Redding, and Weinstein (2016).

${ }^{10}$ In other words, we are assuming that $b_{f i d}=b_{f j d}$ and that $g_{f i}\left(w_{c}\right)$ does not depend on $i$. In Appendix C3, we generalize the analysis to allow the rising marginal cost of products that are farther from the core-competency of the firm. If we restrict
} 
fixed costs to differ across firms. Then the profit maximization problem (13) is simplified as:

$$
\max _{p_{f i d}, N_{f d} \geq 0} \sum_{d=1}^{D}\left\{N_{f d}\left[\left(p_{f i d}-g_{f}-T_{c d}\right) x_{f i d}-k_{f d}\right]-K_{f d}\right\} \mathbf{1}\left(N_{f d}>0\right) .
$$

where $x_{f i d}=x_{f j d}$ when $p_{f i d}=p_{f j d}$. The optimal price is still given by (14), though now with symmmetry this price is the same across varieties $i$ for each firm in city $d$. As the firm expands the number of varieties sold, it draws demand away from existing varieties. Taking this cannibalization effect into account, it is shown in Appendix C.2 that the optimal variety is determined by,

$$
N_{f d}=\frac{\eta-1}{\sigma-1}\left[\frac{S_{f d}\left(1-S_{f d}\right)}{\eta-(\eta-1) S_{f d}}\right] \frac{Y_{d}}{k_{f d}}
$$

where $Y_{d}=\rho w_{d} L_{d}$ is the total expenditure in destination $d$. Substituting this equation into (15) and also using the optimal price from (14), it can be shown that the profits from entering a city, before deducting the fixed costs $K_{f d}$, are: ${ }^{11}$

$$
\pi_{f d}=\frac{(\sigma-\eta)+(\eta-1) S_{f d}}{\sigma-1}\left[\frac{S_{f d}}{\eta-(\eta-1) S_{f d}}\right] Y_{d}
$$

\subsection{Firm Entry}

In order for the firm to serve city $d$, we must have $\pi_{f d} \geq K_{f d}$. To see how city size affects the entry of firms into cities, consider comparing a large city $c$ with a smaller city $d$, with $Y_{d}<Y_{c}$. An equilibrium consists of a set of firms $F_{c}$ and $F_{d}$ selling in each city such that prices for each firm are given by (14), revenue per product is given by (11), and the number of products is as in (16), so that $S_{f c}=N_{f c} p_{f i c} x_{f i c} / Y_{c}$ with $\pi_{f c} \geq K_{f c}$ and $\sum_{f \in F_{c}} S_{f c}=1$, and likewise in city $d$. There is ambiguity in the equilibrium set of firms that enter each city, because once a firm enters then demand for other firms is reduced. But regardless of this ambiguity, we assert that if $Y_{d}<Y_{c}$ then there exists a weakly smaller set of entrants $F_{d} \subseteq F_{c}$ in city $d$ satisfying the equilibrium conditions, and that the entrants are definitely reduced if city $d$ is small enough.

To establish this claim, start with the equilibrium conditions for city $c$ and then reduce expenditure $Y_{c}$. From (16) we see that $N_{f c}$ falls in direct proportion to $Y_{c}$ provided that $S_{f c}=N_{f c} p_{f i c} x_{f i c} / Y_{c}$ is not affected. In fact, that is the equilibrium result because it can be confirmed from (11) that with all firms selling fewer products in direct proportion to the fall in expenditure $Y_{c}$, then the change in the CES price indexes $P_{f_{c}}$ and $P_{c}$ ensure that revenue per product is not affected. Profits in (17) will fall in direct proportion to the fall in $Y_{c}$, however. It follows that for a sufficiently large reduction in expenditure, the firm with the smallest initial ratio $\pi_{f c} / K_{f c} \geq 1$ will be the first to have $\pi_{f c} / K_{f c}<1$, and will therefore exit the market. It follows that $F_{d} \subset F_{c}$, as we asserted.

Denoting the firm that exits by $g$, the equilibrium condition $\sum_{f \in F_{c}} S_{f c}=1$ will become $\sum_{f \in F_{c} \backslash g} S_{f c}=$ 1 , so the market shares of all remaining firms will rise as that firm drops out (because expenditure

the analysis to iceberg rather than specific trade costs, then we find that the equilibrium condition for the scope of a firm is essentially the same as that shown by (16), but with an extra constant term.

${ }^{11}$ To derive (17) we use that fact that the revenue earned per product by firm $f$ is $S_{f d} Y_{d} / N_{f d}$. 
$p_{f i c} x_{f i c}$ on all remaining products increases as the price index $P_{c}$ rises in (11)). By this argument, we see that smaller cities will have fewer firms in our model, with higher market shares. That will lead to higher prices in (14), since we have assumed that marginal costs for each firm are the same across cities. In other words, there is an anti-competitive effect in smaller cities, or a pro-competitive effect in larger cities. We now turn to the empirical analysis to find whether this prediction is borne out in the data for Chinese and U.S. cities.

\section{Estimating the Firm Model}

We will estimate (14) by moving the markup term to the left, so that the remaining marginal costs plus transport costs on the right will be estimated:

$$
\begin{aligned}
p_{f i d}\left[1+\frac{1}{(\eta-1)\left(1-S_{f d}\right)}\right]^{-1} & =g_{f i}\left(w_{c}\right)+T_{c d} \\
& =\alpha_{i}+\alpha_{c a p}+\beta \ln \operatorname{Dist}_{c d}+\epsilon_{f i d} .
\end{aligned}
$$

The first term on the right is an indicator variable for variety $i$, which together with the eror term $\epsilon_{\text {fid }}$ reflects the marginal cost of production. We include an indictor variable $\alpha_{c a p}$ for the capital city of each province in China, and the $\log$ of distance $\ln D i s t_{c d}$ between the production in city $c$ and the destination city $d .{ }^{12}$

Let $N_{f, \min }=\min _{c}\left\{N_{f c}\right\}$ denote the "common" varieties sold by firm $f$ in all cities within each country. Then the common-goods share of firm $f$ in destination $d$ is:

$$
\lambda_{f d}=\frac{N_{f, \min } p_{f d} x_{f d}}{N_{f d} p_{f d} x_{f d}}=\frac{N_{f, \min }}{N_{f d}}=N_{f, \min }\left(\frac{\sigma-1}{\eta-1}\right)\left[\frac{\eta-(\eta-1) S_{f d}}{S_{f d}\left(1-S_{f d}\right)}\right] \frac{k_{f d}}{Y_{d}},
$$

using (16). The second regression equation comes from substituting $Y_{d}=\rho w_{d} L_{d}$ and taking $\log$,

$$
\ln \lambda_{f d}=\gamma_{f, p r o v}+\delta_{1} \ln \left[\frac{\eta-(\eta-1) S_{f d}}{S_{f d}\left(1-S_{f d}\right)}\right]+\delta_{2} \ln w_{d}+\delta_{3} \ln L_{d}+\epsilon_{f d} .
$$

The first term $\gamma_{f \text {, prov }}$ on the right is an indicator variable for the firm-province, which together with the eror term $\epsilon_{f d}$ reflects variation in $N_{f, \min }$ and in the fixed costs $k_{f d}$ of providing each product in city $d$. The second term reflects an inverted-U-shaped relationship between the product scope of a firm and its market share $S_{f d}$, as shown by (16). Small firms have low shares and low scope, but large firms with high shares will hold back on product scope to avoid cannibalizing their own sales; product scope is maximized for an intermediate value of the firm share. ${ }^{13}$ The common-goods share of firms in each city, $\lambda_{f d}$, is inversely related to their product scope, so the second term on the right of (20) is a U-shaped function of the firm's market share, and should have a coefficient of $\delta_{1}=1$ in theory.

\footnotetext{
${ }^{12}$ We have checked via internet sources that the firms in our sample have only one manufacturing location for Toothpaste, Laundry Detergent, Personal Wash items and Shampoo.

${ }^{13}$ In fact, product scope $N_{f d}$ in (16) is maximized for $S_{f d}=\sqrt{\eta} /(1+\sqrt{\eta})$.
} 
Notice that the presence of $w_{d}$ and $L_{d}$ in (20) reflects the fact that higher expenditure in larger cities reduces the common-goods share of firms in that city, $\lambda_{f d}$, thereby increasing product variety from each firm. In theory, the coefficients of these variables are both $\delta_{2}=\delta_{3}=-1$. A negative sign on these estimated coefficients will show how larger cities (measured by population or average income) have a lower common-goods share for firms, and therefore more product variety. We will likewise want to evaluate how prices charged by firms differ across cities of different sizes, and for this purpose we include the variables $w_{d}$ and $L_{d}$ in (18), so that we actually estimate,

$$
p_{f i d}\left[1+\frac{1}{(\eta-1)\left(1-S_{f d}\right)}\right]^{-1}=\alpha_{i}+\alpha_{c a p}+\beta_{1} \ln D i s t_{c d}+\beta_{2} \ln w_{d}+\beta_{3} \ln L_{d}+\epsilon_{f i d} .
$$

\subsection{Estimating the Pro-Competitive Effect}

Our first testable hypothesis is the existence of the pro-competitive effect, which is evaluated with the price equation as shown in (21). For each of the four products, we use both the price $(p)$ and the price divided by the markup ( $p /$ markup) as the dependent variables. We use the scraped prices at the barcode level and rely on Nielsen (China) data to calculate firm-destination shares and the markups. Information on city populations and average incomes, used to measure $Y_{c}=\rho w_{c} L_{c}$, are obtained for China and for MSAs in the United States, from standard sources. Finally, we used company reports to identify the factory locations in China, and therefore compute the distance between the factory and destination markets.

Table 1 shows the result for toothpaste. We use price-per-item (U.S. cents/item) as the dependent variable for specification (1) and (2), and unit-price (U.S. cents/oz) for specification (3) and (4). The coefficients of ln Population and ln Average Income are all significantly negative, which implies larger and richer cities will benefit from lower prices for a given product at the barcode level. Notice that in specification (1), the coefficient on $\ln$ Population falls by about one-third, from -2.87 to -1.92 , when we switch from using the price as the dependent variable to using the price divided by markup. This

finding implies a higher markup in smaller cities, as the markup-adjusted price (price divided by markup) rises less in smaller cities than the per-item price.

We repeat the regressions but controlling for retailer (or supermarket) fixed effects in specification (2). The finding of higher markup in smaller and poorer cities remains robust. Interestingly, the coefficient on ln Population increases from -2.87 to -4.13 after controlling for supermarket fixed effects, i.e. comparing specifications (1) and (2) using the price as the dependent variable, and then again falls by about one-third to -2.68 when using price divided by the markup. This means that even within retailers, we find that firms charge lower prices in larger cities. The results in specification (2) continue to support the presence of a pro-competitive effect, even after controlling for the supermarket. 
Table 1: Price Regressions for Toothpaste

\begin{tabular}{lcccccccc}
\hline \hline & \multicolumn{2}{c}{$(1)$} & \multicolumn{2}{c}{$(2)$} & \multicolumn{2}{c}{$(3)$} & \multicolumn{2}{c}{$(4)$} \\
Dep Var. & Price & P/mkup & Price & P/mkup & Unit-P & UP/mkup & Unit-P & UP/mkup \\
\hline \multirow{3}{*}{ ln Population } & $-2.87^{* * *}$ & $-1.92^{* * *}$ & $-4.13^{* * *}$ & $-2.68^{* * *}$ & $-0.61^{* * *}$ & $-0.40^{* * *}$ & $-0.94^{* * *}$ & $-0.60^{* * *}$ \\
& $(0.25)$ & $(0.18)$ & $(0.24)$ & $(0.15)$ & $(0.07)$ & $(0.05)$ & $(0.06)$ & $(0.04)$ \\
In Average Income & $-3.99^{* * *}$ & $-3.32^{* * *}$ & $-5.95^{* * *}$ & $-4.54^{* * *}$ & $-0.90^{* * *}$ & $-0.69^{* * *}$ & $-1.35^{* * *}$ & $-0.98^{* * *}$ \\
& $(0.32)$ & $(0.24)$ & $(0.31)$ & $(0.20)$ & $(0.08)$ & $(0.06)$ & $(0.08)$ & $(0.06)$ \\
Capital City & $3.59^{* * *}$ & $2.15^{* * *}$ & $1.16^{* * *}$ & $0.65^{* * *}$ & $0.85^{* * *}$ & $0.52^{* * *}$ & $0.27^{* * *}$ & $0.16^{* * *}$ \\
& $(0.26)$ & $(0.17)$ & $(0.22)$ & $(0.14)$ & $(0.07)$ & $(0.04)$ & $(0.06)$ & $(0.04)$ \\
ln Distance & $0.75^{* * *}$ & $0.49^{* * *}$ & $1.07^{* * *}$ & $0.67^{* * *}$ & $0.18^{* * *}$ & $0.12^{* * *}$ & $0.27^{* * *}$ & $0.17^{* * *}$ \\
& $(0.18)$ & $(0.10)$ & $(0.17)$ & $(0.11)$ & $(0.05)$ & $(0.03)$ & $(0.05)$ & $(0.03)$ \\
& & & & & & & & \\
\hline Barcode FE & YES & YES & YES & YES & YES & YES & YES & YES \\
Retailer FE & & & YES & YES & & & YES & YES \\
Observations & 89,399 & 89,399 & 89,399 & 89,399 & 89,399 & 89,399 & 89,399 & 89,399 \\
Number of group & 1,758 & 1,758 & 21,614 & 21,614 & 1,758 & 1,758 & 21,614 & 21,614 \\
R-squared & 0.006 & 0.009 & 0.015 & 0.019 & 0.005 & 0.006 & 0.010 & 0.012 \\
\hline \hline
\end{tabular}

Notes: Average Income is measured as GDP per capita in each city of the given Population. Prices (Price and P/mkup) are in units of U.S. cents and unit-prices (Unit-P and UP/mkup) are measured in U.S. cents per ounce. Capital City is dummy variable. Robust standard errors are clustered at the group level and reported in parentheses; ${ }^{* * *} \mathrm{p}<0.01,{ }^{* *}$ $\mathrm{p}<0.05,{ }^{*} \mathrm{p}<0.1$.

Specifications (3) and (4) use the unit-price (U.S. cents/oz) as the dependent variable. We observe similar results as in specifications (1) and (2), and the pro-competitive effect remains significant: the coefficients of $\ln$ Population and $\ln$ Average Income are uniformly negative, and the coefficients are reduced when using unit-price/markup as a dependent variable rather than just unit-price.

In Table 2, we explore the pro-competitive effect for the other product categories (Laundry Detergent, Personal Wash items and Shampoo). We focus on the price regressions with per-item price and price/markup as the dependent variables, but the results using unit-price are similar. From these tables, we still observe that in richer and bigger cities prices are lower and that firms charge a lower markup, as shown by the smaller coefficients on the $\ln$ Population and $\ln$ Average Income when using the using the price divided by markup as the dependent variable. For all four products, therefore, we confirm a pro-competitive effect. ${ }^{14}$

\footnotetext{
${ }^{14}$ Campbell and Hopenhayn (2005) also find this result for thirteen retail trade industries across 225 U.S. cities.
} 
Table 2: Price Regressions for the Other Product Categories

\begin{tabular}{lcccccc}
\hline \hline & \multicolumn{2}{c}{ (1) Laundry Detergent } & \multicolumn{2}{c}{ (2) Personal Wash } & \multicolumn{2}{c}{ (3) Shampoo } \\
Dep Var. & Price & P/mkup & Price & P/mkup & Price & P/mkup \\
\hline \multirow{3}{*}{ In Population } & $-3.69^{* * *}$ & $-2.72^{* * *}$ & $-1.47^{* * *}$ & $-1.37^{* * *}$ & $-4.88^{* * *}$ & $-3.44^{* * *}$ \\
& $(0.41)$ & $(0.30)$ & $(0.33)$ & $(0.23)$ & $(0.48)$ & $(0.37)$ \\
ln Average Income & $-4.24^{* * *}$ & $-3.12^{* * *}$ & $-4.86^{* * *}$ & $-3.48^{* * *}$ & $-6.50^{* * *}$ & $-5.68^{* * *}$ \\
& $(0.45)$ & $(0.33)$ & $(0.50)$ & $(0.35)$ & $(0.63)$ & $(0.49)$ \\
Capital City & $0.86^{* *}$ & $0.65^{* *}$ & 0.45 & 0.29 & 0.67 & 0.18 \\
& $(0.42)$ & $(0.31)$ & $(0.37)$ & $(0.25)$ & $(0.53)$ & $(0.41)$ \\
ln Distance & $1.14^{* * *}$ & $0.84^{* * *}$ & $1.66^{* * *}$ & $1.09^{* * *}$ & $3.65^{* * *}$ & $2.85^{* * *}$ \\
& $(0.30)$ & $(0.22)$ & $(0.33)$ & $(0.23)$ & $(0.39)$ & $(0.30)$ \\
& & & & & & \\
\hline Observations & 109,650 & 109,650 & 159,673 & 159,673 & 142,826 & 142,826 \\
Number of group & 28,001 & 28,001 & 35,965 & 35,965 & 31,711 & 31,711 \\
R-squared & 0.003 & 0.003 & 0.002 & 0.002 & 0.005 & 0.005 \\
\hline \hline
\end{tabular}

Notes: All regressions include Barcode and Retailer fixed effects. Average Income is measured as GDP per capita in each city of the given Population. Prices (Price and P/mkup) are in units of U.S. cents. Capital City is dummy variable. Robust standard errors are clustered at the group level and reported in parentheses; ${ }^{* * *} \mathrm{p}<0.01,{ }^{* *} \mathrm{p}<0.05,{ }^{*} \mathrm{p}<0.1$.

\subsection{Estimating the Variety Effect}

Table 3 exhibits the result of our second structural equation as shown in (20). Motivated by the model, we add brand-province fixed effects to control for the fixed costs needed to sell each variety in each city, $k_{f d}$. The dependent variable is $\ln \lambda_{f c}$, which denotes the expenditure in city $c$ on the common products of firm $f$ relative to firm $f^{\prime}$ s total sales in that city. Columns (1) to (4) report results for Toothpaste, Laundry Detergent, Personal Wash items, and Shampoo respectively. The regressions results show that larger and richer cities are likely to have smaller expenditure shares on common products for a given firm. This result implies that larger and richer cities get access to more varieties than smaller cities. Consistent with model, the variable $\ln \frac{\eta-(\eta-1) S_{f d}}{S_{f d}\left(1-S_{f d}\right)}$ contributes to the common product share positively, but with a coefficient less than its theoretical value of unity.

These empirical results support the inverted U-shaped relation between product scope and market share in (16), from which the regression specification (20) is obtained. Feenstra and Ma (2009) showed that in a model with heterogeneous, multiproduct firms, an inverted U-shaped relation would hold between the productivity of the firm and its product scope: more productive firms initially add more products, but then reduce product scope as cannibalization becomes more important. Raff and Wagner (2013) have shown that such an inverted U-shaped relation holds empirically in a sample of German firms, and Macedoni (2017) obtains this relation in more general theoretical and empirical settings. Our results here are therefore consistent with these authors. 
Table 3: Firm Share Regression

\begin{tabular}{lcccc}
\hline \hline & $(1)$ & $(2)$ & $(3)$ & $(4)$ \\
Dep Var: $\ln \lambda_{f d}$ & Toothpaste & Laundry Detergent & Personal Wash & Shampoo \\
\hline \multirow{3}{*}{$\ln$ Population } & $-0.209^{* * *}$ & $-0.236^{* * *}$ & $-0.295^{* * *}$ & $-0.156^{* * *}$ \\
& $(0.037)$ & $(0.030)$ & $(0.034)$ & $(0.021)$ \\
$\ln$ Average Income & $-0.059^{* *}$ & $-0.244^{* * *}$ & $-0.159^{* * *}$ & $-0.083^{* * *}$ \\
& $(0.029)$ & $(0.030)$ & $(0.030)$ & $(0.012)$ \\
Capital City & $-0.125^{* * *}$ & $-0.179^{* * *}$ & $-0.136^{* * *}$ & $-0.069^{* * *}$ \\
& $(0.023)$ & $(0.029)$ & $(0.028)$ & $(0.015)$ \\
$\ln \frac{\eta-(\eta-1) S_{f d}}{S_{f d}\left(1-S_{f d}\right)}$ & $0.147^{* * *}$ & $0.609^{* * *}$ & $0.396^{* * *}$ & 0.002 \\
& $(0.033)$ & $(0.070)$ & $(0.061)$ & $(0.006)$ \\
& & & & \\
\hline Observations & 660 & 420 & 600 & 840 \\
Number of group & 308 & 196 & 280 & 392 \\
R-squared & 0.376 & 0.662 & 0.586 & 0.348 \\
\hline \hline
\end{tabular}

Notes: All regressions include firm-province fixed effects. Average Income is measured as GDP per capita in each city of the given Population. Capital City is dummy variable. Robust standard errors are clustered at group level and reported in parentheses; ${ }^{* * *} \mathrm{p}<0.01,{ }^{* *} \mathrm{p}<0.05,{ }^{*} \mathrm{p}<0.1$.

\section{Extension to other Products}

\subsection{Measuring Product Variety}

We turn now to the additional products for which we collected scraped data on their retail prices in 60 cities, but for which we do not have any scanner data purchased from Nielsen (China). This means that we do not have any information on their market shares, beyond knowing how many supermarkets in each city sell each product (as is evident from the scraped data). In addition, as noted earlier, the United States and China use different barcode systems (UPC and EAN-13, respectively). For that reason, it is desirable to develop formulae for the exact price indexes that do not rely on having identical taste parameters $b_{f i c}$ for common goods that are available in every city and country. We now show how the CES index derived by Redding and Weinstein (2016) allows for a much weaker assumption on taste parameters that allows the cost of living to be compared between countries.

To briefly review the results of Redding and Weinstein (2016), we start with the demand for each product variety, which equals $b_{f i c} x_{f i c}=\left[\left(p_{f i c} / b_{f i c}\right) / P_{f c}\right]^{-\sigma} X_{f c}$. Multiplying by $\left(p_{f i c} / b_{f i c}\right)$ and dividing by $P_{f c} X_{f c}$, we obtain an equation for the share of each variety within the total sales of firm $f$, which depends on the CES price index $P_{f_{c}}$. Inverting that equation to solve for $P_{f_{c}}$, we readily obtain:

$$
P_{f c}=e\left(\mathbf{p}_{f c}, \mathbf{b}_{f c}, I_{f c}\right)=s_{f i c}^{1 /(\sigma-1)}\left(\frac{p_{f i c}}{b_{f i c}}\right)
$$


The term $\lambda_{f c}$ defined in (6) equals $s_{f i c} / s_{f i c}\left(I_{f}\right)$, so we can replace the share $s_{f i c}$ by $s_{f i c}=s_{f i c}\left(I_{f}\right) \lambda_{f c}$ in the above equation. Then because (22) holds for every product $i$, we take the unweighted geometric mean across all products to obtain the formula in Redding and Weinstein (2016):

$$
P_{f c}=\left[\prod_{i \in I_{f}} s_{f i c}\left(I_{f}\right)^{\frac{1}{N_{f}(\sigma-1)}}\right]\left[\prod_{i \in I_{f}}\left(\frac{p_{f i c}}{b_{f i c}}\right)^{\frac{1}{N_{f}}}\right] \lambda_{f c}^{1 /(\sigma-1)} .
$$

Notice that this formula gives us the level of the CES price index $P_{f_{c}}$, and not just its ratio as shown in section 2 by equation (7).

We can aggregate over firms in a city using a similar approach. The aggregate demand for each firm's products are $X_{f_{c}}=\left(P_{f_{c}} / P_{c}\right)^{-\eta}\left(Y_{c} / P_{c}\right)$. Multiplying by $P_{f_{c}}$ and dividing by $Y_{c}$, we obtain the share of each firm in city $c$, which depends on the CES price index $P_{c}$. Inverting that equation to solve for $P_{c}$, we readily obtain:

$$
P_{c}=E\left(\mathbf{P}_{c}, F_{c}\right)=S_{f c}^{1 /(\eta-1)} P_{f_{c}} .
$$

We again replace the share $S_{f_{c}}$ by $S_{f_{c}}=S_{f_{c}}(F) \lambda_{c}$, and take the unweighted geometric mean over the number of common firms $M$ selling to all cities in each country. Then using that geometric mean along with (23), we obtain:

$$
P_{c}=E\left(\mathbf{P}_{c}, F_{c}\right)=\left[\prod_{f \in F} \prod_{i \in I_{f}}\left(\frac{p_{f i c}}{b_{f i c}}\right)^{\frac{1}{M N_{f}}}\right] S_{c}(F) \Lambda_{c}(F)
$$

where,

$$
S_{c}(F) \equiv\left[\prod_{f \in F} S_{f c}(F)^{\frac{1}{M(\eta-1)}} \prod_{i \in I_{f}} s_{f i c}\left(I_{f}\right)^{\frac{1}{M N_{f}(\sigma-1)}}\right], \Lambda_{c}(F) \equiv\left[\prod_{f \in F} \lambda_{f c}^{1 / M(\sigma-1)}\right] \lambda_{c}^{1 /(\eta-1)} .
$$

The first expression on the right of (24) is a simple geometric mean of the prices of common products, but these prices are adjusted for the taste parameters $b_{f i c}$. We have earlier assumed in our discussion of (7) that the taste parameters $b_{\text {fic }}$ are identical for common goods that are available in every city within each country. We can now follow the assumption that Redding and Weinstein (2016) make in a time-series context, and use the weaker assumption that the geometric mean of taste parameters are equal in every city within each country:

$$
\prod_{f \in F} \prod_{i \in I_{f}}\left(b_{f i c}\right)^{\frac{1}{M N_{f}}}=1, \quad \forall c \in=1, \ldots, D .
$$

With this assumption, the first term on the right of (24) is easily measured as the geometric mean of prices. The second term $S_{c}(F)$ is a geometric mean of the common firms and and common product shares, as shown in (25). We interpret that term as adjusting for differences in tastes for specific 
products within the common set, but subject to the maintained assumption that average tastes are the same as in (26). The third term $\Lambda_{c}(F)$ is the correction for variety outside the common set as in Feenstra (1994).

We see that (24) and (25) can be readily used to measure the CES price index $P_{c}$ across all cities within each country. But what about when we compare China and the United States? In that case, we need some analogue to the assumption in (26) that we are willing to apply across countries. To develop this idea, let us denote the global brands by the name of the common firms that sell in both countries by the set $G \equiv F^{U S} \cap F^{\text {China }}$, which we assume is not empty. ${ }^{15}$ We then pick the two large and comparable cities in each country, New York (NY) and Shanghai (SH). Our assumption is that there has been enough "convergence" of tastes and availability of global brands across these two cities so that the average taste parameters are the same,

$$
\prod_{g \in G} \prod_{i \in I_{g}}\left(b_{g i, N Y}\right)^{\frac{1}{M N_{g}}}=\prod_{g \in G} \prod_{i \in I_{g}}\left(b_{g i, S H}\right)^{\frac{1}{M N_{g}}} .
$$

With this assumption, we can compare the CES price indexes $P_{c}$ between countries, as follows. We first use (24) to measure the CES price index $P_{c}$ for New York in U.S. and Shanghai in China, with the common international firms $G$, which are denoted as $E\left(\mathbf{P}_{N Y}, G\right)$ and $E\left(\mathbf{P}_{S H}, G\right)$. Next, we apply the same calculations to the cost-of-living measures across all cities in China, with the common firms $F^{\text {China }}$, and all cities in the United States, with the common firms $F^{U S}$. We then renormalize the Chinese and U.S. price indexes so that the cost-of-living indexes are the same for Shanghai and New York when using the common set as the global brands $(G)$ and national brands ( $F^{\text {China }}$ and $F^{U S}$ ). This is achieved by computing the terms in (24) for the global brands $G$ only in New York and in Shanghai, and then renormalizing the same terms for the Chinese and U.S. sets of common product $F^{\text {China }}$ and $F^{U S}$ as:

$$
\begin{aligned}
\widetilde{E}\left(\mathbf{P}_{c}, F_{c}^{\text {China }}\right) & \equiv E\left(\mathbf{P}_{c}, F_{c}^{\text {China }}\right) \times \frac{E\left(\mathbf{P}_{S H}, G\right)}{E\left(\mathbf{P}_{S H}, F_{S H}^{\text {China }}\right)} \\
\widetilde{E}\left(\mathbf{P}_{c}, F_{c}^{U S}\right) & \equiv E\left(\mathbf{P}_{c}, F^{U S}\right) \times \frac{E\left(\mathbf{P}_{N Y}, G\right)}{E\left(\mathbf{P}_{N Y}, F_{N Y}^{U S}\right)}
\end{aligned}
$$

Since this renormalization is done for all cities in both countries, we obtain cost-of-living indexes that are fully comparable between cities and countries.

\subsection{Cost-of-Living Indexes for the Four Main Products}

We calculate the cost-of-living indexes in New York and Shanghai, making use of assumption (27) so that these indexes are comparable between countries. The renormalization in (28) has already been

\footnotetext{
${ }^{15}$ The global brands by product categories are listed in Table 9 in Appendix A4. In practice, $G$ is empty for Chips and we alternatively define $G \equiv\left[F^{U S} \cap\left(\cup F_{c}^{\text {China }}\right)\right] \cup\left[F^{\text {China }} \cap\left(\cup F_{c}^{U S}\right)\right]$, the global firms that are either sell national common products in China or in the U.S., and mark it with asterisk.
} 
applied to the indexes in panel (c) of Figure 2 to 5, which are otherwise constructed according to the method of Feenstra (1994). For comparison, we also calculate the indexes for these four products according to the method of Redding and Weinstein (2016), which uses the unweighted geometric mean of prices in (24), denoted by $P_{c}^{G}$, together with the multiplied terms $S_{c}(F) \Lambda_{c}(F)$. Those indexes are illustrated in panel (d) of Figures 2 to 5, which are also renormalized according to (28).

A glance at those earlier Figures shows that the negative relationship between the exact price index and city size is noticably weaker, especially for Toothpaste, when using the Redding-Weinstein (RW) index than when using the Feenstra index. To formalize that difference, in Tables 4 and 5 we report the coefficients of regressions of the exact price indexes and its components on the log of city size (measured now with city GDP). Specifically, we run the regressions:

$$
\ln Z_{c}=\mu_{1}+\mu_{c a p}+\mu_{2} \ln Y_{c}+\epsilon_{d}
$$

where $Z_{c}$ denotes either of the two terms in (10) or any of the three terms on the right of (24). Table 4 uses the Feenstra exact index in (10) and its components, while Table 5 uses the RW index in (24) and its components.

Table 4: Decomposition of Cost of living with City Size (Feenstra Method)

\begin{tabular}{lcccccccc}
\hline \hline & \multicolumn{2}{c}{ Toothpaste } & \multicolumn{2}{c}{ Laundry Detergent } & \multicolumn{2}{c}{ Personal Wash } & \multicolumn{2}{c}{ Shampoo } \\
Dep. $\downarrow \backslash$ Indep. $\rightarrow$ & $\ln Y_{c}$ & $R^{2}$ & $\ln Y_{c}$ & $R^{2}$ & $\ln Y_{\mathcal{c}}$ & $R^{2}$ & $\ln Y_{c}$ & $R^{2}$ \\
\hline $\ln E\left(P_{f_{c}}, F_{c}\right)$ & $-0.122^{* * *}$ & 0.424 & $-0.044^{* * *}$ & 0.419 & $-0.081^{* * *}$ & 0.320 & $-0.029^{* * *}$ & 0.233 \\
& $(0.022)$ & & $(0.007)$ & & $(0.018)$ & & $(0.007)$ & \\
& & & & & & & & \\
$\ln P_{c}^{S V}$ & $-0.055^{* * *}$ & 0.363 & -0.003 & 0.051 & $-0.021^{* *}$ & 0.122 & $-0.015^{*}$ & 0.095 \\
& $(0.010)$ & & $(0.006)$ & & $(0.008)$ & & $(0.007)$ & \\
$\ln \Lambda_{c}^{S V}$ & $45.1 \%$ & & $6.8 \%$ & & $25.6 \%$ & & $51.7 \%$ & \\
& $-0.067^{* *}$ & 0.361 & $-0.041^{* * *}$ & 0.524 & $-0.061^{* * *}$ & 0.395 & -0.014 & 0.079 \\
& $(0.015)$ & & $(0.006)$ & & $(0.013)$ & & $(0.009)$ & \\
& $51.9 \%$ & & $93.2 \%$ & & $74.4 \%$ & & $48.3 \%$ & \\
\hline \hline
\end{tabular}

Notes: All regressions also include Capital dummy variable and constant, which are omitted in the table. $P_{\mathcal{C}}^{S V}$ denotes the Sato-Vartia weighted mean of unit prices, $\Lambda_{c}^{S V}$ is the variety term in (10), and $Y_{c}$ is city GDP. Standard errors are reported in parentheses; ${ }^{* * *} \mathrm{p}<0.01,{ }^{* *} \mathrm{p}<0.05,{ }^{*} \mathrm{p}<0.1$.

For Toothpaste, we see that the coefficients of the Feenstra exact index, and its components using Sato-Vartia weights, are more than twice as sensitive to city size than the RW exact index, and its geometric price and variety components. The percentage contribution of these regression coefficients shows that the negative relationship between the exact price index and city size is caused roughly equally by the negative relationship between price and variety with city size. For example, out of the 
regression coefficient of the price index on $\ln Y_{c}$ of -0.122 in the first column of Table 4, around -0.055 (or $45.1 \%$ ) is explained by the regression of the Sato-Vartia index $\ln P_{c}^{S V}$ on $\ln Y_{c}$, while about -0.067 (or $51.9 \%$ ) is explained by the regression of $\ln \Lambda_{c}^{S V}$ on $\ln Y_{c}^{S V}$.

Similar calculations are done in the other columns in Table 4, and in Table 5 which now also includes the term $\ln S_{c}(F)$. That final variable appearing in Table 5 is is either insignficantly related to city size or has a very small coefficient. Thus, it is not the presence of that share variable in the RW index that accounts for its reduced sensitivity to city size. But this variable will have a very important impact on the average level of the exact price index in China as compared to the U.S., especially as we turn to the other 15 products that we consider next.

Table 5: Decomposition of Cost of living with City Size (Redding-Weinstein Method)

\begin{tabular}{lcccccccc}
\hline \hline & \multicolumn{2}{c}{ Toothpaste } & \multicolumn{2}{c}{ Laundry Detergent } & \multicolumn{2}{c}{ Personal Wash } & \multicolumn{2}{c}{ Shampoo } \\
Dep. $\downarrow \backslash$ Indep. $\rightarrow$ & $\ln Y_{\mathcal{c}}$ & $R^{2}$ & $\ln Y_{\mathcal{c}}$ & $R^{2}$ & $\ln Y_{\mathcal{c}}$ & $R^{2}$ & $\ln Y_{\mathcal{c}}$ & $R^{2}$ \\
\hline $\ln E\left(P_{f c}, F_{c}\right)$ & $-0.045^{* * *}$ & 0.166 & $-0.051^{* * *}$ & 0.453 & $-0.042^{* * *}$ & 0.357 & -0.014 & 0.069 \\
& $(0.016)$ & & $(0.008)$ & & $(0.008)$ & & $(0.007)$ & \\
& & & & & & & & \\
$\ln P_{c}^{G}$ & $-0.025^{* * *}$ & 0.185 & -0.006 & 0.056 & -0.004 & 0.058 & -0.006 & 0.149 \\
& $(0.009)$ & & $(0.006)$ & & $(0.005)$ & & $(0.005)$ & \\
$\ln \Lambda_{c}(F)$ & $55.6 \%$ & & $11.8 \%$ & & $9.5 \%$ & & $42.9 \%$ & \\
& $-0.027^{* *}$ & 0.261 & $-0.037^{* * *}$ & 0.478 & $-0.059^{* * *}$ & 0.363 & -0.010 & 0.054 \\
& $(0.009)$ & & $(0.006)$ & & $(0.014)$ & & $(0.009)$ & \\
$\ln S_{c}(F)$ & $60.0 \%$ & & $72.5 \%$ & & $140.5 \%$ & & $71.4 \%$ & \\
& 0.007 & 0.090 & $-0.008^{*}$ & 0.094 & 0.021 & 0.121 & 0.002 & 0.027 \\
& $(0.017)$ & & $(0.003)$ & & $(0.013)$ & & $(0.003)$ & \\
& $-15.6 \%$ & & $15.7 \%$ & & $-50 \%$ & & $-14.3 \%$ & \\
\hline \hline
\end{tabular}

Notes: All regressions also include Capital dummy variable and constant, which are omitted in the table. $P_{c}^{G}$ denotes the geometric mean of unit prices, $\Lambda_{c}(F)$ is the variety term, $S_{c}(F)$ is the share term in (25), and $Y_{c}$ is city'GDP. Standard errors are reported in parentheses; ${ }^{* * *} \mathrm{p}<0.01,{ }^{* *} \mathrm{p}<0.05,{ }^{*} \mathrm{p}<0.1$.

\subsection{Cost-of-Living Indexes for 15 Other Product Categories}

When we have only scraped price data and not the Nielsen (China) barcode expenditures, it is challenging to the construct the share term $S_{c}(F)$ and also the variety term $\Lambda_{c}(F)$ that appears in in (24). We create a proxy for the expenditure on each barcode product by simply counting the number of retail stores that sell each barcode in a city (and dividing by the total number of retailers). That is, the sales share of each product is approximated by:

$$
\ln \widehat{\text { Share }}_{i c} \propto \frac{\sum_{r \in R_{c}} \mathbf{1}(i \text { is observed in retailer } r)}{N_{c}^{\text {retailers }}}
$$


where $\mathbf{1}(i$ is observed in retailer $r)$ equals unity if we observe price quotes of barcode product $i$ in retailer $r$ and zero otherwise; $R_{c}$ denote the collection of retailers in city $c$; and $N_{c}^{\text {retailers }}$ is the total number of retailer stores in city $c{ }^{16}$

In Table 6, we apply the RW index from (24) and (28) to our four main products and to the other 15 product categories. We summarize each component of the Chinese cost of living relative to the average value of its counterpart of the U.S. A value greater than one implies the corresponding variable is larger in China than in the United States. Beginning with the geometric mean of prices, as shown in the first column, these are lower in China for all products except for Shampoo, Baby Formula, Cereals and Milk Powder.

Table 6: Summary of Cost of Living by Product Categories (relative to the U.S.)

\begin{tabular}{lcccc}
\hline \hline Product Category $\backslash$ Variable & $P_{c}^{G}$ & $\frac{S_{S H}(G) \Lambda_{S H}(G)}{S_{N Y}(G) \Lambda_{N Y}(G)}$ & Other Terms & $\tilde{E}\left(P_{f c}, F_{c}\right)$ \\
\hline & \multicolumn{2}{c}{ Four Main Products } & & \\
Toothpaste & 0.81 & 0.64 & 1.13 & 0.58 \\
Laundry Detergent & 0.59 & 0.52 & 0.76 & 0.23 \\
Personal Wash Item & 0.80 & 0.62 & 0.55 & 0.25 \\
Shampoo & 1.59 & 0.66 & 0.61 & 0.65 \\
& Other Products & & \\
Baby Formula & 1.30 & 1.12 & 1.02 & 1.48 \\
Batteries & 0.24 & 1.01 & 1.27 & 0.31 \\
Biscuits & 0.64 & 2.18 & 3.05 & 4.23 \\
Cereal & 1.29 & 1.95 & 0.70 & 1.76 \\
Chips & 0.31 & 1.76 & 1.16 & 0.64 \\
Chocolate & 0.74 & 1.74 & 1.37 & 1.77 \\
Coffee & 0.70 & 0.97 & 1.00 & 0.68 \\
Diapers & 0.74 & 1.12 & 1.04 & 0.86 \\
Dog Food & 0.23 & 1.46 & 1.06 & 0.35 \\
Gum & 0.93 & 0.88 & 0.82 & 0.67 \\
Milk Powder & 5.29 & 0.74 & 1.60 & 6.20 \\
Soap & 0.37 & 4.28 & 0.89 & 1.43 \\
Soft Drinks & 0.41 & 1.19 & 0.67 & 0.33 \\
Tea & 0.81 & 2.13 & 1.12 & 1.93 \\
Toothbrushes & 0.53 & 1.57 & 1.13 & 0.93 \\
\hline \hline
\end{tabular}

Notes: The table summarizes population weighted average of each varaible in relative terms (greater than unity implies the corresponding variable is larger in China.). $P_{\mathcal{C}}^{G}$ is in unit of cents per ounce for the four main products and dollars per item for other products; $\frac{S_{S H}(G) \Lambda_{S H}(G)}{S_{N Y}(G) \Lambda_{N Y}(G)}$ denotes the composite share of varieties in Shanghai relative to New York; and Other Terms is the further adjustment needed to obtain $\tilde{E}\left(P_{f_{c}}, F_{c}\right)$, which is the cost-of-living index in China relative to the U.S.

\footnotetext{
${ }^{16}$ See Appendix B3 and Antoniades (2017), where it is shown that the average market share is exponentially related to the proportion of available retailers carying the barcode item. It follows that the log of the share is linearly related to this proportion.
} 
In the second column we show the ratio of the terms $\left[S_{S H}(G) \Lambda_{S H}(G) / S_{N Y}(G) \Lambda_{N Y}(G)\right]$ for Shanghai relative to New York. This is the key ratio that adjusts the relative geometric mean of prices to obtain the relative cost of living in these two cities. This ratio is less than unity for our four main products, indicating that there is greater product variety in China than in the United States. We interpret that result as saying that the global brands $G$ are sufficiently common in China for these four products that the presence of additional local brands in China gives it greater product variety.

For the 15 other product categories, however, this ratio is greater than unity for all products except for three: Coffee, Gum and Milk Powder. Coffee and Gum are similar to our four main products, where there are (inexpensive) local brands in China that are sufficient popular to result in greater product variety there. Along with Soft Drinks, which also has local brands in China, these three products in addition to the four main products have cost-of-living indexes in China relative to the U.S. that are lower than the relative geometric mean of prices shown in the first column.

Milk Powder is a unique case because there was a consumer scare about this product due to the Chinese milk scandal in 2008 and now the prices are higher than in the United States, as shown in the first column. Those high prices are slightly offset by a value of $\left[S_{S H}(G) \Lambda_{S H}(G) / S_{N Y}(G) \Lambda_{N Y}(G)\right]$ that is less than unity, but the exact price index shown in the final column is still much higher in China. That feature is shared by all the remaining ten products: the cost of living in China relative to the U.S., as shown in the final column, is higher than the relative geometric mean of prices. That higher cost of living in China for the remaining products is in most cases due to lower variety in China. ${ }^{17}$ In some products (Biscuits, Chocolate, Soap, and Tea), that lower variety is strong enough that it reverses the lower relative price in China, as shown by the geometric mean of prices, to become a higher exact price index in China.

It is surprisingly that there is lower relative variety in China to a product like Tea, since China has specialized in the product from ancient times. What seems to explain the lower relative variety in our data is that the many loose varieties of Chinese tea are barely sold in supermarkets, which is where we obtain our scraped data. So we miss entirely the local varieties of Chinese tea and end up with only Western-style teas, with Lipton as the unique global brand that is sold in all cities in both countries. The finding of a higher relative price of tea in China illustrates a deficiency of our data, or more precisely, of the attempt to correct the prices of barcode products for variety. It remains to be seen whether other methods of obtaining prices and expenditure data across countries can overcome this limitation.

For each of the 15 product categories, we also explore to what extent the three components (denoted by $P_{c}^{G}, S_{c}(G)$ and $\left.\Lambda_{c}(G)\right)$ contribute to the overall cost of living. Table 7 presents the regression results of the three components on city size (i.e. city GDP). ${ }^{18}$. The first column is the elasticity of the cost-of-living index with respect to the city size. As expected, larger cities generally benefit from having a lower cost-of-living index. Cities with twice the market size have lower indexes with the

\footnotetext{
${ }^{17}$ We further decompose $\left[S_{S H}(G) \Lambda_{S H}(G) / S_{N Y}(G) \Lambda_{N Y}(G)\right]$ into three margins as shown in the Appendix D, Table 11.

${ }^{18}$ In each regression, we also include a dummy variable for the capital city which is not reported in the Table.
} 
declines ranging from $1 \%$ (Chocolate) to $10.4 \%$ (Cereal). Pro-competitive effects are operative in most cases, as shown by the negative coefficients in the second column, with the exception of Batteries, Biscuits, Chocolate, Milk Powder, Soap and Toothbrushes. The final two columns show the relative importance of the share term $S_{c}(G)$ and the variety term $\Lambda_{c}(G)$ in explaining the lower cost of living in larger cities in China. In most cases, it is the variety term that is the most important explanation for the lower exact price index and therefore the gains from living in larger cities.

Table 7: Decomposition of Cost of living with City Size (Other Product Categories)

\begin{tabular}{|c|c|c|c|c|}
\hline Product & $\ln \tilde{E}\left(P_{f_{c}}, F_{c}\right)$ & $\ln P_{c}^{G}$ & $\ln S_{c}(G)$ & $\ln \Lambda_{c}(G)$ \\
\hline \multirow[t]{2}{*}{ Baby Formula } & -0.014 & -0.008 & -0.003 & -0.003 \\
\hline & & $59 \%$ & $20 \%$ & $21 \%$ \\
\hline \multirow[t]{2}{*}{ Batteries } & -0.017 & 0.005 & -0.013 & -0.009 \\
\hline & & $-27 \%$ & $76 \%$ & $51 \%$ \\
\hline \multirow[t]{2}{*}{ Biscuits } & -0.028 & 0.001 & 0.002 & -0.030 \\
\hline & & $-2 \%$ & $-7 \%$ & $109 \%$ \\
\hline \multirow[t]{2}{*}{ Cereal } & -0.104 & -0.020 & -0.013 & -0.071 \\
\hline & & $19 \%$ & $13 \%$ & $68 \%$ \\
\hline \multirow[t]{2}{*}{ Chips } & -0.019 & -0.011 & 0.008 & -0.016 \\
\hline & & $57 \%$ & $-44 \%$ & $87 \%$ \\
\hline \multirow[t]{2}{*}{ Chocolate } & -0.010 & 0.003 & 0.005 & -0.019 \\
\hline & & $-33 \%$ & $-51 \%$ & $184 \%$ \\
\hline \multirow[t]{2}{*}{ Coffee } & -0.054 & -0.012 & 0.003 & -0.045 \\
\hline & & $23 \%$ & $-6 \%$ & $83 \%$ \\
\hline \multirow[t]{2}{*}{ Diapers } & -0.020 & -0.015 & 0.001 & -0.006 \\
\hline & & $76 \%$ & $-5 \%$ & $29 \%$ \\
\hline \multirow[t]{2}{*}{ Dog Food } & -0.069 & -0.014 & -0.020 & -0.035 \\
\hline & & $20 \%$ & $29 \%$ & $51 \%$ \\
\hline \multirow[t]{2}{*}{ Gum } & -0.029 & -0.012 & -0.008 & -0.010 \\
\hline & & $41 \%$ & $26 \%$ & $33 \%$ \\
\hline \multirow{2}{*}{ Milk Powder } & -0.049 & 0.007 & 0.012 & -0.068 \\
\hline & & $-14 \%$ & $-24 \%$ & $138 \%$ \\
\hline \multirow[t]{2}{*}{ Soap } & -0.033 & 0.008 & -0.015 & -0.026 \\
\hline & & $-23 \%$ & $45 \%$ & $78 \%$ \\
\hline \multirow[t]{2}{*}{ Soft Drinks } & -0.072 & -0.022 & 0.003 & -0.053 \\
\hline & & $31 \%$ & $-4 \%$ & $73 \%$ \\
\hline \multirow[t]{2}{*}{ Tea } & -0.057 & -0.006 & -0.013 & -0.038 \\
\hline & & $10 \%$ & $23 \%$ & $67 \%$ \\
\hline \multirow[t]{2}{*}{ Toothbrushes } & -0.080 & 0.005 & -0.026 & -0.059 \\
\hline & & $-6 \%$ & $32 \%$ & $74 \%$ \\
\hline
\end{tabular}

Notes: The reported coefficients denote the elasticity with respect to the total market size. All regressions also include Capital dummy variable and constant, which are omitted in the table. Percentages describe the contribution of each margin to the overall cost-of-living elasticity. $P_{\mathcal{c}}^{G}$ denotes the geometric mean of unit prices, $\Lambda_{\mathcal{c}}(G)$ is the variety term and $S_{c}(G)$ is the share term in (25), for the global brands $G$. 


\section{Conclusions}

Anecdotal evidence and price data collected by the World Bank in 2005 under the International Comparison Program (ICP) suggest that prices in China may be higher than expected. While part of the issue is related to sampling (Feenstra, Ma, Neary, and Prasada Rao (2013)) and procedural decisions made by the World Bank in linking regions with each other (Deaton and Aten (2017), Inklaar and Rao (2017)), there is still an open question as to what prices in China really are.

To address this question, and to overcome limitations in the ICP data, we have used barcode data on selected consumer goods to compare the cost of living in cities across and between China and the U.S. Specifically, we have obtained from Nielsen price and quantity information on Toothpaste, Laundry Detergent, Personal wash items and Shampoo sold in 22 cities across China, and combined those with additional price data on grocery-store products sold in sixty cities that we scraped from a phone application to compute cost-of-living indexes. We also used the Nielsen Retailing Sales (U.S.) database to calculate the cost of living in the U.S.

Our main finding is that the cost-of-living for grocery-store products in China is lower in larger cities. This is driven by two sources: more product variety in large cities and lower prices. The finding that product prices in large Chinese cities are lower contrasts with evidence from the U.S. that show the opposite relation (Handbury and Weinstein (2015)). We attribute lower prices to a procompetitive effect, whereby large cities attract more firms which leads to lower markups and prices. Greater variety in larger cities applies to the U.S., too, but it is more pronounced in China.

We further compare the cost-of-living indexes for particular product groups between China and the United States, which is the goal of the ICP. Other cross-country work by Cavallo and Rigobon (2016) uses a simple geometric mean of barcode level prices to compare price levels. It is noteworthy that the recent theory by Redding and Weinstein (2016), described in section 6, also uses a simple geometric mean of prices. In order to obtain an exact price index, that geometric mean is adjusted by two other terms: a term reflecting the firm shares of products within the geometric mean; and a term reflecting the expenditures shares on unique products in each city, that are not included in the geometric means and which indicate product variety as in Feenstra (1994).

The simple geometric mean of prices is lower in China than in the United States for most product categories: exceptions include Shampoo, Baby Formula, Cereal and Powdered Milk. By focusing on "global brands" to construct these geometric means, we can plausibly meet the theoretical restriction in Redding and Weinstein (2016) that the average tastes across barcode items in these global brands are the same in the two countries. It turns out that focusing on the global brands also reduces the differences in the firm shares, one of the terms adjusting the simple geometric means. ${ }^{19}$

The main difference between the geometric mean and the exact price index, therefore, comes

\footnotetext{
${ }^{19}$ The term $S_{c}(F)$ shown in (25) is a geometric mean of the common firm shares $S_{f_{c}}(F)$ and and common product shares $s_{f i c}\left(I_{f}\right)$. If there is only one global firm, for example, then $S_{g c}(G)=1$ so it does not vary across cities or countries. Appendix D shows the variation in $S_{c}(G)$ and $\Lambda_{c}(G)$ for Shanghai and New York across product categories, as an indication of where the variation in the exact price indexes between these two cities arises from.
} 
from the product variety term. The magnitude of these products variety terms in China relative to the U.S. differs across the products in our sample. In the four product categories for which we purchased Nielsen (China) scanner data, i.e., Toothpaste, Laundry Detergent, Personal Wash items and Shampoo, the availability of additional Chinese brands leads to greater variety than in the United States, and therefore lower Chinese price indexes for that reason. In the other 15 products for which we only have scraped data, however, there is much less presence of U.S. brands in the Chinese market. In these cases, the observed prices differences between the countries (usually lower prices in China) are partially or fully offset by the variety differences (less variety in China), so that the cost of living in China is not as low as the price differences suggest. This latter case applies to Tea, for example, because our data do not include the many Chinese varieties of tea that are not sold in supermarkets. So we miss entirely the local varieties of Chinese tea and end up with only Westernstyle teas, with Lipton as the unique global brand of tea that is sold in all cities in both countries. This finding of a (supposed) higher exact price index for tea in China illustrates a limitation of our attempt to correct the prices of barcode products for variety, particularly when we only have supermarket information. It remains to be seen whether other methods of obtaining price and expenditure data across countries, while correcting for variety, can overcome this limitation. 


\section{Appendix}

\section{A. Collection Method for Retail Price Data of China}

Chinese retail price data are collected by scraping data from a mobile phone application. The mobile phone app that we rely on is Wochacha (the English meaning is "I search"), a leading consumerproduct information platform in China.

\section{A1. Introduction to Wochacha}

The mobile phone application Wochacha was developed by Wochacha Info Tech Co. Ltd in January 2010. Detailed information regarding Wochacha can be obtained from its web-page. ${ }^{20}$ It is a widely used price comparison app, using a QR code scanner so that people can see where a particular product can be bought at the lowest price. Consumers can also search for price quotes based on the barcode of the product. ${ }^{21}$ By February 2014, the number of users had exceeded 210 million and are distributed across all the provinces in China.

According to its Public Service Rules, Wochacha uses two methods to collect the retail price data. First, the firm hires and trains data collectors who visit supermarkets to collect prices. The second source of retail price data relies on partnerships with supermarkets, from which Wochacha directly obtains the scanner data. In either method, the collected prices are not published until Wochacha data auditors have examined them.

\section{A2. Data Collection Example}

Figure 10 exhibits the results when one search for prices quotes of Pampers ${ }^{22}$ in Shanghai. Based on the results, there are eight retail price quotes varying from 112RMB in Walmart to $165 R M B$ in Lotus. Besides the price-by-supermarket information, we also observe the producer name, country of origin, as well as the unit count and producer ID. We developed software to mimic the mobile phone app on the computer to automatically download price data for the given set of EAN codes in the selected city. Then we repeated this process across 60 sampled cities to construct our retailing price database. The collection took place from October to December in 2015.

\section{A3. Cities for Analysis}

Cities for which we scraped price data are listed in Table 8. We include 60 cities from 28 provinces, which are widely spread across China. As shown in Figure 11, many small cities are included in our analysis.

\footnotetext{
${ }^{20}$ See http://www.wochacha.com/about/index.

${ }^{21}$ Countries like U.S. and Canada use Universal Product Code (UPC) as the unique identity for products, whereas China adopts the coding system of European Article Number which technically refers to EAN-13.

${ }^{22}$ The EAN-13 is 6903148040737.
} 

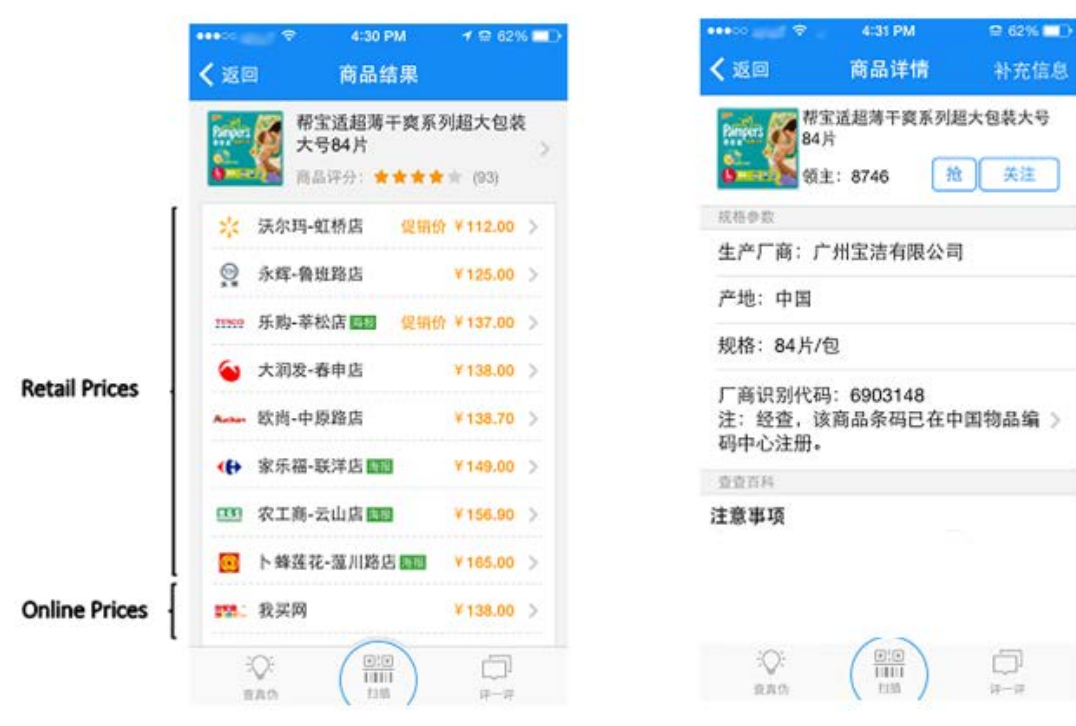

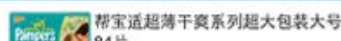

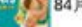

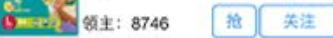

arkop

生产厂商：广州宝洁有限公司

Producer Name

产地: 中国

Country of Origin

规格: 84 片/包

Unit Count

厂竞识别代码: 6903148

注: 经楂, 该商品条码已在中国物品缩

Producer ID 注的。

골ำ

注意事项

Figure 10: Interface of Wochacha Application

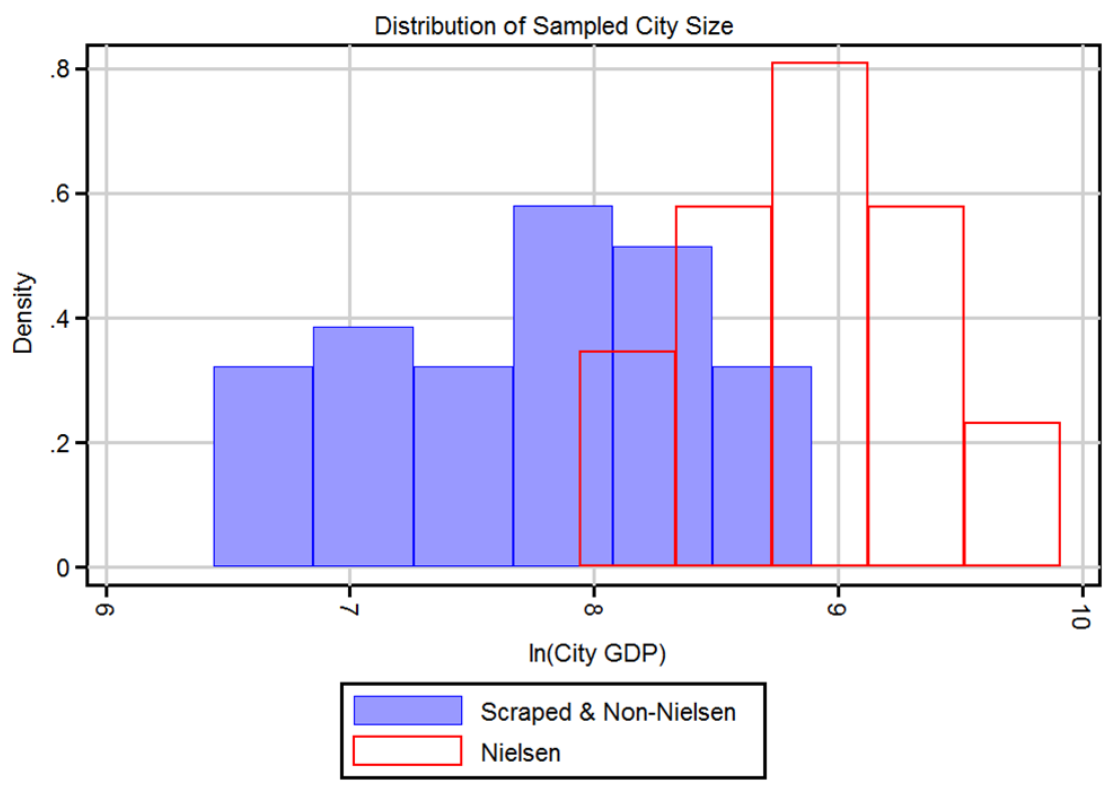

Figure 11: City Size Distribution of the Sampled Cities 


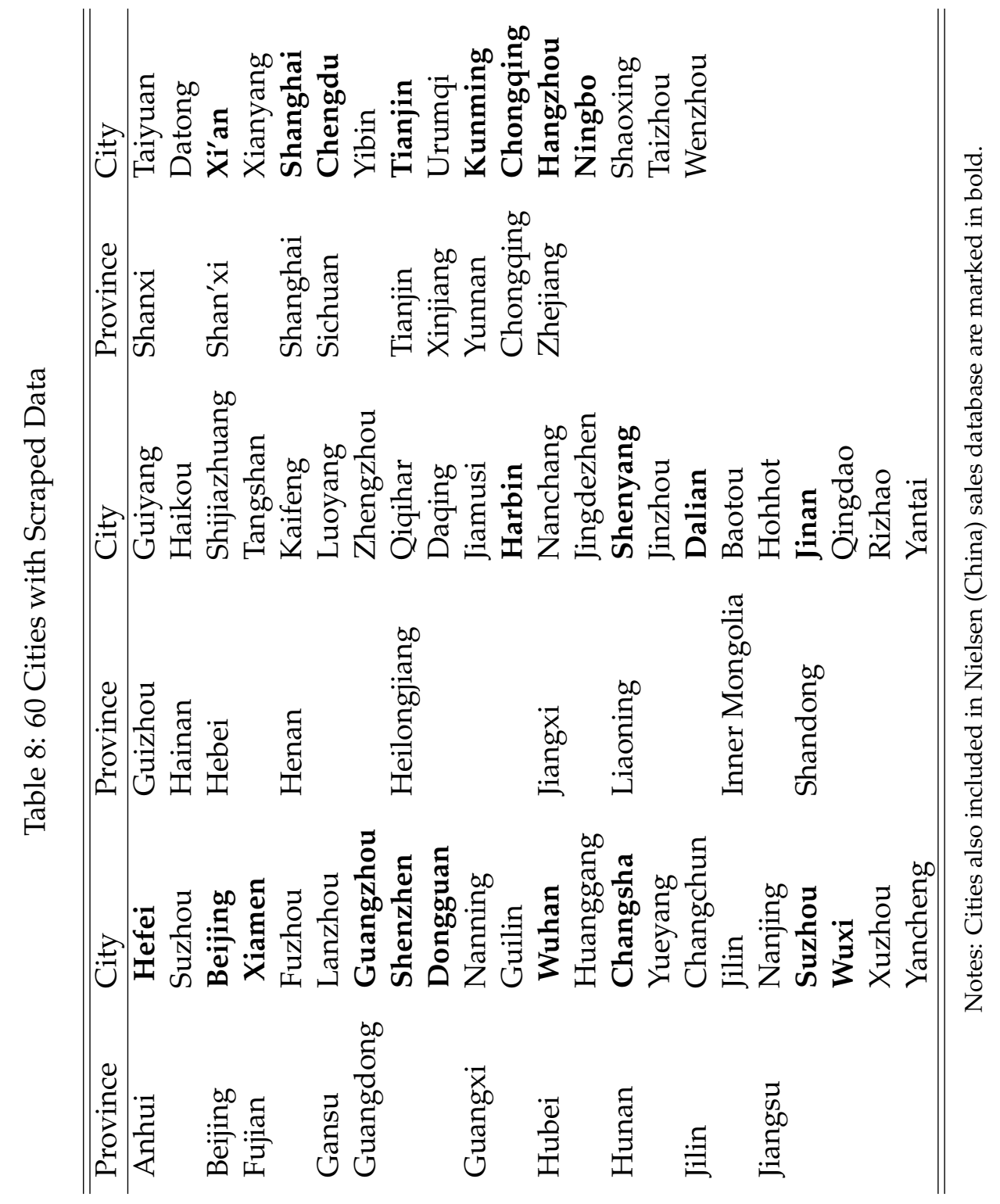




\section{A4. Global Brands}

The global brands for each product are listed in Table 9. For all products except Chips, the global brands are computed as the intersection of the common brands in the U.S. and in China, $G \equiv F^{U S} \cap$ $F^{\text {China }}$. For Chips this set is empty, so we adopt the broader definition of global brands as $G \equiv$ $\left[F^{U S} \cap\left(\cup F_{c}^{\text {China }}\right)\right] \cup\left[F^{\text {China }} \cap\left(\cup F_{c}^{U S}\right)\right]$.

Table 9: List of Global Brands by Product

\begin{tabular}{llll}
\hline \hline Product Category & Global Brands & Product Category & Global Brands \\
\hline Toothpaste & Colgate & Chocolate & M\&M \\
& Crest & Coffee & Maxwell \\
& Sensodyne & Diapers & Huggies \\
Shampoo & Dove & & Pampers \\
& L'Oreal & Dog Food & Pedigree \\
Personal Wash Items & Pantene & Gum & Wrigley \\
Laundry Detergent & Tide & Milk Powder & Nestle \\
Baby Formula & Enfamil & Soap & Johnson \\
& Nestle & & Olay \\
& Similac & Soft Drinks & Safeguard \\
Batteries & Duracell & & Minute Maid \\
& GP & Tea & Red Bull \\
Biscuits & Teiji & Toothbrushes & Colgate \\
Cereal & Quaker & & Crest \\
Chips & Doritos & & \\
& Kellogg & & \\
& Lay'S & & \\
& Oishi & & \\
\hline \hline
\end{tabular}

\section{B. Other Data}

\section{B1. Elasticities of Substitution}

We use the elasticities of substitution for our four main products (Toothpaste, Laundry Detergent, Personal Wash items and Shampoo) as well as for other product categories from Hottman, Redding, and Weinstein (2016), which are listed in Table 10. 
Table 10: Elasticity of Substitution

\begin{tabular}{lcc}
\hline \hline Product Category & Firm Elasticity $(\eta)$ & Product Elasticity $(\sigma)$ \\
\hline Toothpaste & 2.75 & 5.23 \\
Laundry Detergent & 3.85 & 6.60 \\
Personal Wash & 3.42 & 5.11 \\
Shampoo & 4.43 & 6.54 \\
Baby Formula & 7.47 & 14.62 \\
Batteries & 8.72 & 15.10 \\
Biscuits & 5.51 & 7.31 \\
Cereal & 2.25 & 4.66 \\
Chips & 4.37 & 7.32 \\
Chocolate & 5.13 & 7.44 \\
Coffee & 3.89 & 7.31 \\
Diapers & 7.44 & 24.30 \\
Dog Food & 5.51 & 8.15 \\
Gum & 3.14 & 4.86 \\
Milk Powder & 2.41 & 5.05 \\
Soap & 3.42 & 5.11 \\
Soft Drinks & 5.18 & 7.82 \\
Tea & 4.46 & 6.12 \\
Toothbrushes & 2.75 & 5.23 \\
\hline \hline
\end{tabular}

\section{B2. Prediction Method in Four Main Product Categories}

The cost of living calculation requires firm sales information, whereas the Nielsen (China) sales database only provide us with this information for only 22 large cities. To allow our analysis to also cover smaller cities, we estimate the market share by using the product, retailer and city information for the four main products, as discussed in this part.

For the four main products, we have the detailed EAN-level market sales in 22 cities, in addition to the price quotes collected by scraping the mobile phone application. We implement a Heckman two-step approach to estimate the product share within the 22 cities using the Nielsen (China) sales database, as shown below:

$$
\begin{gathered}
\operatorname{Prob}_{\left(\text {Share }_{i c}>0\right)}=\alpha_{0}+\alpha_{1} \ln Y_{c}+\alpha_{2} \ln L_{c}+\alpha_{3} \ln N_{c}+\alpha_{4} \text { Capital }_{c} \\
+\delta_{i} \text { Variety }_{i}+\gamma_{1 r} \text { Region }_{r}+\epsilon_{i c}
\end{gathered}
$$

where (31) is the selection equation and (32) is the observation equation. $Y_{c}$ denotes city c's GDP; $L_{c}$ is city population; $N_{c}$ denotes the total number of differentiated products (at EAN level) in city $c$; Capital $_{c}$ is a dummy variable which equals unity if city $c$ is a capital city; Region ${ }_{r}$ is a set of region 
dummies (East, Middle and West China). Finally, for each product $i$ at EAN-13 level, Brand $i$ denotes a collection of Brand dummies, e.g., Crest, Colgate etc. for Toothpaste.; Variety $y_{i}$ is a collection of variety categories which is constructed by jointly combining several product characteristics ${ }^{23}$; Share $_{i c}$ is calculated as product $i$ 's sales share in city $c$. The two-step Heckman approach is motivated by the fact that we only observe price quotes in the mobile phone application conditional on that product is sold in a particular city. To reduce the endogeneity issues in price regression, we do not include the product price on the right of the share specification (32).

After obtaining the coefficients $(\hat{\alpha}, \hat{\beta}, \hat{\gamma}, \hat{\delta}, \hat{\phi}, \hat{\mu}, \hat{\psi})$, we predict the market share in the cities that do not have sales information, and we further rescale them so that the sum of all product shares equals unity within each city. For consistency, we use the predicted shares to calculate the cost-of-living index for all cities, including the 22 for which we have the expenditure data from Nielsen (China).

Though this prediction method works well in most cities, it results in extreme predictions in some cities, particularly when some product accounts for $80 \%$ or more of the total market. To avoid this situation, we exclude cities that have the extremely low city's average firm sales share of common products $\prod_{f \in F}\left(\lambda_{f c}\right)^{W_{f}(F)}$ from our analysis. Specifically, we firstly regress city's average firm sales share of common products, $\lambda_{c}^{F} \equiv \prod_{f \in F}\left(\lambda_{f c}\right)^{W_{f}(F)}$, on city's population (ln $\left.L_{c}\right)$, and derived the prediction error $\hat{u}_{c}=\lambda_{c}^{F}-\hat{\lambda}_{c}^{F}$. Then we drop the cities from our sample if its prediction error is twice larger than the standard deviation of the prediction errors, i.e., we exclude city $c$ if $\left|\hat{u}_{c}\right|>2 \times \operatorname{std}(\hat{u})^{24}$. After implementing the criteria, we finally have (out of 60 cities) 58 cities for Toothpaste, Laundry Detergent and Personal Wash Items, and 57 cities for Shampoo.

\section{B3. Prediction Method for Other Products}

Consider a world where there are $N$ distinct barcode products and $M$ number of homogeneous retailers. For demonstration purpose, we assume the products can be classified into two broad categories that differ in availability (this assumption is for simplicity, while in reality it could be many). Specifically, the probability of observing the appearance of any $H$-type and $L$-type products in any retailer are $a_{H}$ and $a_{L}$ respectively. Denote the share of $H$-type and $L$-type products are $s_{H}$ and $s_{L}=1-s_{H}$. As all the $\left\{a_{H}, a_{L}, s_{H}, s_{L}\right\}$ are unobservable in data, we use expected share of a given product to approximate the real market share for both products. The expected market share of products that are observed in $k$ outlet is Share $_{k}$ that is given as:

$$
\text { Share }_{k}=s_{H}\left(\begin{array}{c}
M \\
k
\end{array}\right) a_{H}^{k}\left(1-a_{H}\right)^{M-k}+s_{L}\left(\begin{array}{c}
M \\
k
\end{array}\right) a_{L}^{k}\left(1-a_{L}\right)^{M-k}
$$

where $\left(\begin{array}{c}M \\ k\end{array}\right)$ denotes the number of combinations of $M$ choose $k$. It is immediate that the average market share is in exponential relationship with the proportion of the outlets selling that product

\footnotetext{
${ }^{23}$ For example, for Toothpaste, we define a variety by brand, subbrand, special function, flavors, and form. Variety Crest-Complete Plus-Deep Clean-Mint-Paste is different from Crest-Complete Plus-Mouth Wash-Mint-Paste

${ }^{24} \mathrm{We}$ also tried with one and three standard deviations, and the main results barely changed.
} 
(especially there are many types of products). To demonstrate this, we propose a numerical example where we set $a_{H}=0.95, a_{L}=0.8, s_{H}=0.6, s_{L}=0.4$ and $M=10$, and plot the relationship bewteen Share $_{k}$ and $k / M$ as shown below:

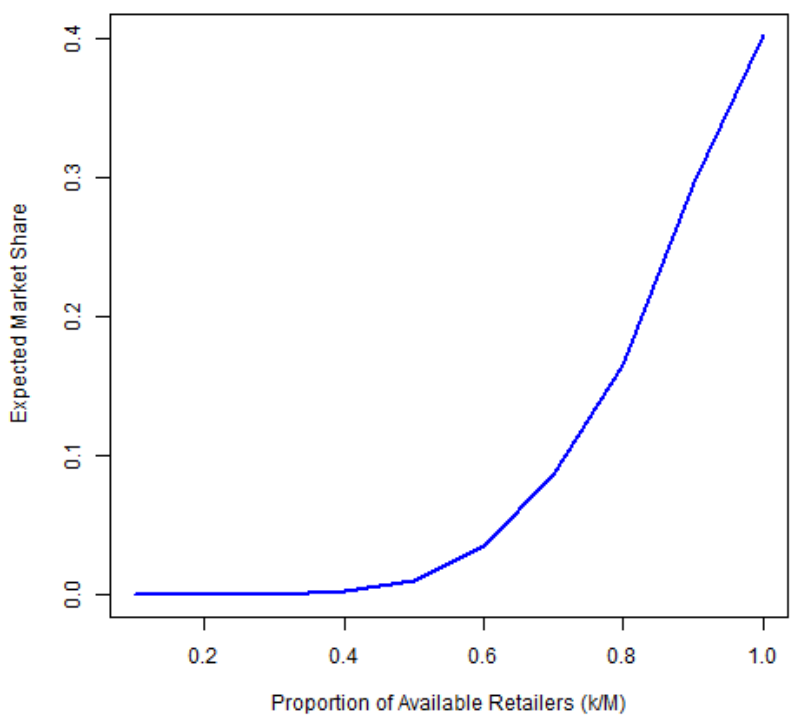

Figure 12: The Expected Market Share of Products Available in $k$ Retail Outlets

Because of the exponential relationship between $S_{h a r e}$ and $k / M$, we take the natural log to obtain equation (30) in the main text. ${ }^{25}$

\section{Solution to the Firm's Problem}

\section{C1. Choice of price by the firm}

The solution to the problem (13) w.r.t. the price $p_{\text {fid }}$ is

$$
x_{f i d}+\sum_{j \in I_{f d}}\left[p_{f j d}-g_{f j}\left(w_{c}\right)-T_{c d}\right] \frac{d x_{f j d}}{d p_{f i d}}=0, \quad \forall i \in I_{f d} .
$$

This expression is more complicated than for a single-product firm because the firm is selling all the products $i \in I_{f d}$, and therefore must take into account the effect of a change in each price $p_{f i c}$ on all these products. To simplify this expression, it can be confirmed that the CES demand derivatives are symmetric, $d x_{f j d} / d p_{f i d}=d x_{f i d} / d p_{f j d}$. Using (34) and dividing by demand $x_{f i d}$, we can re-express the condition as

$$
1+\sum_{j \in I_{f d}}\left[1-\frac{g_{f j}\left(w_{c}\right)+T_{c d}}{p_{f j d}}\right] \frac{d \ln x_{f i d}}{d \ln p_{f j d}}=0, \quad \forall i \in I_{f d}
$$

\footnotetext{
${ }^{25}$ The exponential relationship between the average market share and the proportion of the available retailers is empirically found in Antoniades (2017).
} 
Let us denote the ratio of price to marginal cost, inclusive of transport costs, by $\mu_{f j d}=p_{f j d} /\left(g_{f j}+\right.$ $\left.T_{c d}\right) \geq 1$. We can see that the expression in brackets in (35) equals $\left(\mu_{f j d}-1\right) / \mu_{f j d} \geq 0$, which is the difference between price and marginal cost measured relative to price. This is the 'Lerner index' of monopoly power for a single-product firm, and with price chosen optimally will equal the inverse of its elasticity of demand. To see how this Lerner pricing rule is modified with multiproduct firms, let us conjecture a solution where the price-cost ratios are constant across all products sold by the firm in question, $\mu_{f j d}=\mu_{f d}$. Then it is immediately clear that the solution to (35) is

$$
\left(\frac{\mu_{f d}-1}{\mu_{f d}}\right)=-\left(\sum_{j \in I_{f d}} \frac{d \ln x_{f i d}}{d \ln p_{f j d}}\right)^{-1} .
$$

Expression (36) says that the Lerner index for the firm equals the inverse of the sum of demand elasticities (rather than just the inverse of the own demand elasticity, as occurs for a single-product firm). In order for this solution to be valid, however, we need to have that the sum of elasticities on the right of (36) be independent of good $i$, because we have assumed that the markup is common across goods. This independence holds if an equi-proportional increase in all prices charged by a firm needs to lead to the same percentage drop in demand for any product sold by that firm. It turns out that this condition is satisfied for CES demands, in which case the sum of elasticities is ${ }^{26}$

$$
-\sum_{j \in I_{f d}} \frac{d \ln x_{f i d}}{d \ln p_{f j d}}=\eta-(\eta-1) S_{f d}>1 .
$$

Notice that the expression on the right-hand side of (37) is precisely what we get from (12) if we replace the share $s_{f i d}$ within the firm by unity, since the firm sells all of its own products. Then the pricing formula is obtained as (14).

\section{C2. Choice of variety by the firm}

The first-order condition of (15) with respect to $N_{f d}$ yields ${ }^{27}$

$$
\left[p_{f d}-g_{f}\left(w_{c}\right)-T_{c d}\right] x_{f d}+N_{f d}\left[p_{f d}-g_{f i}\left(w_{c}\right)-T_{c d}\right] \frac{d x_{f d}}{d N_{f d}}=k_{f d} .
$$

Using the demand function, the elasticity of demand w.r.t. product scope $N_{f d}$ is given by:

$$
\begin{aligned}
\frac{d \ln x_{f d}}{d \ln N_{f d}} & =(\sigma-\eta) \frac{d \ln P_{f d}}{d \ln N_{f d}}+(\eta-1) \frac{d \ln P_{d}}{d \ln P_{f d}} \frac{d \ln P_{f d}}{d \ln N_{f d}} \\
& =\frac{\sigma-\eta+(\eta-1) S_{f d}}{1-\sigma} .
\end{aligned}
$$

\footnotetext{
${ }^{26}$ The cross-elasticity is $d \ln x_{f i d} / d \ln p_{f j d}=-\left[(\sigma-\eta)+(\eta-1) S_{f c}\right] s_{f j c}$, for $i \neq j$ and along with (12) then (37) is obtained.

${ }^{27}$ As products have the same marginal cost, it is convenient to omit subscript $i$ in the analysis.
} 
The second inequality comes from $\frac{d \ln P_{f d}}{d \ln N_{f d}}=\frac{1}{1-\sigma}$ when marginal costs are the same. Condition (38) can then be rewritten as (16),

$$
N_{f d}=\frac{\eta-1}{\sigma-1}\left[\frac{S_{f d}\left(1-S_{f d}\right)}{\eta-(\eta-1) S_{f d}}\right] \frac{Y_{d}}{k_{f d}}
$$

\section{C3. Model of core-competency and iceberg trade cost detailed}

In this section, we solve the firm's problem with heterogeneous product marginal cost and iceberg trade cost. The production technology of the firm is characterized by a core competence for product $\omega=0$, and firms are able to adjust the number of varieties with an adaption cost in marginal cost. Specifically, the marginal cost of producing variety $\omega$ is assumed to be monotonically increasing in $\omega$. There is also a bilateral iceberg transportation $\operatorname{cost} \tau_{c d}$ when firm sells to other cities. With these assumptions, the profit maximization of firm $f$ in city $c$ is expressed as,

$$
\max _{N_{f d},\left\{p_{f d}(\omega) \mid \omega \in\left[0, N_{f d}\right]\right\}} \sum_{d=1}^{D}\left\{\int_{0}^{N_{f d}}\left[p_{f d}(\omega)-\tau_{c d} g_{f}(\omega)\right] x_{f d}(\omega) d \omega-k_{f d} N_{f d}-K_{f d}\right\} \mathbf{1}\left(N_{f d}>0\right) .
$$

Following the steps shown in Appendix C.1, the optimal price is:

$$
p_{f d}(\omega)=\left[1+\frac{1}{(\eta-1)\left(1-S_{f d}\right)}\right] \tau_{c d} g_{f}(\omega), \quad \forall \omega \in\left[0, N_{f d}\right]
$$

The first order condition with respect to $N_{f d}$ yields,

$$
\left[p_{f d}\left(N_{f d}\right)-\tau_{c d} g_{f}\left(N_{f d}\right)\right] x_{f d}\left(N_{f d}\right)+\int_{0}^{N_{f d}}\left[p_{f d}(\omega)-\tau_{c d} g_{f}(\omega)\right] \frac{d \ln x_{f d}(\omega)}{d N_{f d}} d \omega=k_{f d}
$$

Using the demand function, the elasticity of demand with respect to product scope is given by:

$$
\begin{aligned}
\frac{d \ln x_{f d}}{d \ln N_{f d}} & =(\sigma-\eta) \frac{d \ln P_{f d}}{d \ln N_{f d}}+(\eta-1) \frac{d \ln P_{d}}{d \ln P_{f d}} \frac{d \ln P_{f d}}{d \ln N_{f d}} \\
& =\frac{\sigma-\eta+(\eta-1) S_{f d}}{1-\sigma} s_{f d}\left(N_{f d}\right) N_{f d} .
\end{aligned}
$$

In this expression, $s_{f d}\left(N_{f d}\right)$ denotes the within firm share of the very last product, indexed by $\omega=$ $N_{f d}$, which is defined as,

$$
s_{f d}\left(N_{f d}\right)=\frac{p_{f d}\left(N_{f d}\right) x_{f d}\left(N_{f d}\right)}{\int_{0}^{N_{f d}} p_{f d}(\omega) x_{f d}(\omega) d \omega}=\frac{g_{f}\left(N_{f d}\right)^{1-\sigma}}{\int_{0}^{N_{f d}} g_{f}(\omega)^{1-\sigma} d \omega} \equiv H\left(N_{f d}\right),
$$

where $H($.$) increases with its argument given g_{f}^{\prime}>0$. Jointly using (41) and (42), the optimal scope is derived as,

$$
H\left(N_{f d}\right)=\frac{\eta-1}{\sigma-1}\left[\frac{S_{f d}\left(1-S_{f d}\right)}{\eta-(\eta-1) S_{f d}}\right] \frac{Y_{d}}{k_{f d}} .
$$


If we further assume $g_{f}(\omega)=w_{c} \omega^{\theta}$, with $\theta \geq 0$ and $\sigma<1+1 / \theta$, then $H\left(N_{f d}\right)=(1+\theta-\sigma \theta) / N_{f d}$. Then the equation for $\lambda_{f d}$ is derived as,

$$
\lambda_{f d}=\frac{N_{f d}^{(\sigma-1) \theta} N_{m i n}^{1+\theta-\theta \sigma}}{1+\theta-\theta \sigma}\left(\frac{\sigma-1}{\eta-1}\right)\left[\frac{\eta-(\eta-1) S_{f d}}{S_{f d}\left(1-S_{f d}\right)}\right] \frac{k_{f d}}{Y_{d}} .
$$

\section{Summary of Decomposition of $S(G) \Lambda(G)$ for New York and Shanghai}

Table 11 summarizes the population weighted average of the share term $S_{c}(G)$ and the variety term $\Lambda_{\mathcal{C}}(G)$, which are components of the exact price index shown in (25), for Shanghai relative to New York (greater than unity implies the corresponding variable is larger in Shanghai).

Table 11: Summary of Decomposition of $S(G) \Lambda(G)$ for New York and Shanghai

\begin{tabular}{|c|c|c|c|c|c|}
\hline Product Category $\backslash$ Variable & $\begin{array}{l}\prod_{f \in G} S_{f, S H}(G) \\
\prod_{f \in G} S_{f, N Y}(G)\end{array}$ & $\begin{array}{l}\prod_{f \in G} \prod_{i \in I_{f}} s_{f i, S H}\left(I_{f}\right) \\
\prod_{f \in G} \prod_{i \in I_{f}} s_{f i, N Y}\left(I_{f}\right)\end{array}$ & $\frac{S_{S H}(G)}{S_{N Y}(G)}$ & $\frac{\Lambda_{S H}(G)}{\Lambda_{N Y}(G)}$ & $\frac{S_{S H}(G) \Lambda_{S H}(G)}{S_{N Y}(G) \Lambda_{N Y}(G)}$ \\
\hline \multicolumn{6}{|c|}{ Four Main Products } \\
\hline Toothpaste & 1.01 & 1.53 & 1.55 & 0.41 & 0.64 \\
\hline Laundry Detergent & 1.00 & 1.13 & 1.13 & 0.46 & 0.52 \\
\hline Personal Wash Item & 1.00 & 0.60 & 0.60 & 1.03 & 0.62 \\
\hline Shampoo & 1.06 & 1.05 & 1.11 & 0.60 & 0.66 \\
\hline \multicolumn{6}{|c|}{ Other Products } \\
\hline Baby Formula & 1.25 & 1.01 & 1.27 & 0.88 & 1.12 \\
\hline Batteries & 1.00 & 1.35 & 1.35 & 0.75 & 1.01 \\
\hline Biscuits & 1.00 & 0.98 & 0.98 & 2.23 & 2.18 \\
\hline Cereal & 1.00 & 2.15 & 2.15 & 0.91 & 1.95 \\
\hline Chips & 1.75 & 0.85 & 1.49 & 1.18 & 1.76 \\
\hline Chocolate & 1.00 & 1.62 & 1.62 & 1.07 & 1.74 \\
\hline Coffee & 1.00 & 1.20 & 1.20 & 0.80 & 0.97 \\
\hline Diapers & 1.01 & 1.12 & 1.13 & 0.99 & 1.12 \\
\hline Dog Food & 1.00 & 1.03 & 1.03 & 1.42 & 1.46 \\
\hline Gum & 1.00 & 1.26 & 1.26 & 0.70 & 0.88 \\
\hline Milk Powder & 1.00 & 0.60 & 0.60 & 1.24 & 0.74 \\
\hline Soap & 1.46 & 1.18 & 1.72 & 2.49 & 4.28 \\
\hline Soft Drinks & 1.05 & 1.56 & 1.63 & 0.73 & 1.19 \\
\hline Tea & 1.00 & 1.52 & 1.52 & 1.41 & 2.13 \\
\hline Toothbrushes & 1.99 & 1.33 & 2.65 & 0.60 & 1.57 \\
\hline
\end{tabular}




\section{References}

Antoniades, A. (2017): “Distribution as Expenditure,” Working paper, Georgetown University.

Campbell, J. R., And H. A. Hopenhayn (2005): "Market Size Matters," Journal of Industrial Economics, 53(1), 1-25.

Cavallo, A., And R. Rigobon (2016): “The Billion Prices Project: Using Online Prices for Measurement and Research," Journal of Economic Perspectives, 30(2), 151-178.

DeAton, A., And B. Aten (2017): “Trying to Understand the PPPs in ICP2011: Why are the Results so Different?," American Economic Journal: Macroeconomics, 9(1), 243-264.

Deaton, A., And A. Heston (2010): “Understanding PPPs and PPP-based National Accounts," American Economic Journal: Macroeconomics, 2(4), 1-35.

Feenstra, R. C. (1994): “New Product Varieties and the Measurement of International Prices," American Economic Review, 84(1), 157-177.

(2016): Advanced International Trade,Princeton University Press, 2nd edition.

FEENSTRA, R. C., AND H. MA (2009): “Optimal choice of product scope for multiproduct firms under monopolistic competition," in The Organization of Firms in a Global Economy, ed. by E. Helpman, D. Marin, and T. Verdier, pp. 173-199. Harvard university Press.

Feenstra, R. C., H. Ma, J. P. Neary, and D. Prasada RaO (2013): “Who Shrunk China? Puzzles in the Measurement of Real GDP," The Economic Journal, 123(573), 1100-1129.

Handbury, J., and D. E. Weinstein (2015): “Goods Prices and Availability in Cities," The Review of Economics and Statistics, 82(1), 258-296.

Hottman, C., S. J. Redding, And D. E. Weinstein (2016): “Quantifying the Sources of Firm Heterogeneity," Quarterly Journal of Economics, 131(3), 1291-1364.

INKLAAR, R., AND D. S. P. RAO (2017): “Cross-Country Income Levels Over Time: Did the Developing World Suddenly Become much Richer?," American Economic Journal: Macroeconomics, 9(1), 265-290.

Macedoni, L. (2017): "Multiproduct Superstars Across Rich and Poor Countries. Theory and Evidence," Discussion paper, University of California, Davis.

RAfF, H., And J. WAgner (2013): "Productivity and the Product Scope of Multi-Product Firms: a Test of Feenstra-Ma," Economic Bulletin, 33(1), 173-199.

Redding, S. J., And D. E. Weinstein (2016): “A Unified Approach to Estimating Demand and Welfare," Discussion paper, Princeton University and Columbia University. 
SATO, K. (1976): “The Ideal log-Change Index Number," Review of Economics and Statistics, 58, 223228.

VARTIA, Y. O. (1976): “Ideal log-Change Index Numbers," Scandinavian Journal of Statistics, 3, 121126. 